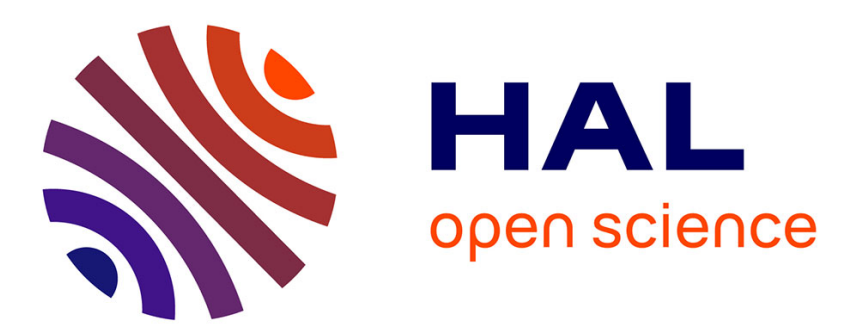

\title{
Bulk properties of the Kondo-like system UCoGe
}

Robert Troc, Ryszard Wawryk, Wojciech Miiller, Halina Misiorek, Malgorzata

Samsel-Czekala

\section{To cite this version:}

Robert Troc, Ryszard Wawryk, Wojciech Miiller, Halina Misiorek, Malgorzata Samsel-Czekala. Bulk properties of the Kondo-like system UCoGe. Philosophical Magazine, 2010, 90 (16), pp.2249-2271. 10.1080/14786431003630900 . hal-00592297

\section{HAL Id: hal-00592297 \\ https://hal.science/hal-00592297}

Submitted on 12 May 2011

HAL is a multi-disciplinary open access archive for the deposit and dissemination of scientific research documents, whether they are published or not. The documents may come from teaching and research institutions in France or abroad, or from public or private research centers.
L'archive ouverte pluridisciplinaire HAL, est destinée au dépôt et à la diffusion de documents scientifiques de niveau recherche, publiés ou non, émanant des établissements d'enseignement et de recherche français ou étrangers, des laboratoires publics ou privés. 


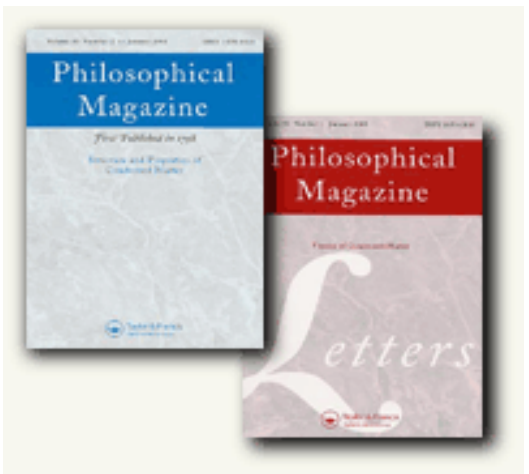

\section{Bulk properties of the Kondo-like system UCoGe}

\begin{tabular}{|c|c|}
\hline Journal: & Philosophical Magazine \& Philosophical Magazine Letters \\
\hline Manuscript ID: & TPHM-09-Sep-0417.R1 \\
\hline Journal Selection: & Philosophical Magazine \\
\hline $\begin{array}{r}\text { Date Submitted by the } \\
\text { Author: }\end{array}$ & 12-Jan-2010 \\
\hline Complete List of Authors: & $\begin{array}{l}\text { Troc, Robert; Institute of Low Temperature and Structure Research } \\
\text { Polish Academy of Sciences, Department of Magnetic Investigation } \\
\text { Wawryk, Ryszard; Institute of Low Temperature and Structure } \\
\text { Research Polish Academy of Sciences } \\
\text { Miiller, Wojciech; Institute of Low Temperature and Structure } \\
\text { Research Polish Academy of Sciences } \\
\text { Misiorek, Halina; Institute of Low Temperature and Structure } \\
\text { Research Polish Academy of Sciences } \\
\text { Samsel-Czekala, Malgorzata; Institute of Low Temperature and } \\
\text { Structure Research Polish Academy of Sciences }\end{array}$ \\
\hline Keywords: & $\begin{array}{l}\text { actinides, superconductors, Kondo effect, thermal properties, } \\
\text { transport properties, magnetoresistance, magnetization, electrical } \\
\text { transport }\end{array}$ \\
\hline Keywords (user supplied): & \\
\hline
\end{tabular}

\section{scholarONE Manuscript Central}




\title{
Bulk properties of the Kondo-like system UCoGe
}

\author{
R. Troć", R. Wawryk, W. Miiller, H. Misiorek, M. Samsel-Czekała \\ Institute of Low Temperature and Structure Research, Polish Academy of Sciences, \\ P.O. Box 1410, 50-950 Wroctaw 2, Poland
}

${ }^{*}$ Author for correspondence. E-mail: r.troc@int.pan.wroc.pl; phone: (+48-71) 3435021 ext.
$\underline{\text { 206; fax: (+48-71) 3441029 }}$

(Received 2009; final version received)

\begin{abstract}
We report on extensive experimental investigation of a single crystal of the orthorhombic uranium compound UCoGe. Bulk measurements being done on as grown and annealed single crystals as magnetization, magnetic susceptibility, electrical resistivity, magnetoresistivity, thermopower, thermal conductivity, and heat capacity data do not reproduce the previously reported coexistence of ferromagnetism with superconductivity. The latter phenomenon was only observed for the annealed sample with $\mathrm{T}_{\mathrm{SC}}=0.65 \mathrm{~K}$. Quite new observations are a crossover at around $13 \mathrm{~K}$ visible in thermal and transport measurements as well as the coherent state around $50 \mathrm{~K}$ signalized by a wide knee in $\rho(\mathrm{T})$. Above this temperature UCoGe exhibits a single-ion Kondo-like effect. The magnetoresistivity of the annealed single crystal is increasing negatively down to $4.2 \mathrm{~K}$, reaching as a large value as about $-27 \%$ at a field of $8 \mathrm{~T}$. The latter may be interpreted in terms of fairly strong magnetic fluctuations existing in $\mathrm{UCoGe}$ at low temperatures.
\end{abstract}

PACS numbers: 74.25.-q, 74.25.Ha, 75.20.Hr, 75.47.-m

\section{Introduction}

The various UTM ternaries ( $T=$ transition metal) with $M=\mathrm{Si}$ and Ge were first prepared by arc melting many years ago [1]. Their orthorhombic crystal structure, mainly of the TiNiSi-type (space group: Pnma) with the $\mathrm{U}$ atoms arranged in characteristic zigzag chains along the $a$ axis, as well as the magnetic properties were recognized. A rule of thumb was found, stating that together with reducing the $\mathrm{d}$ electron occupation the magnetism weakens and magnetic ordering disappears $[1,2]$ Considering only the germanide series, three of them, namely UFeGe [3], UCoGe and URuGe [1] with reduced d-electron occupation have just been found to be paramagnetic down to $4.2 \mathrm{~K}$. The remaining studied compounds in this series, namely 
with $T=\mathrm{Rh}, \mathrm{Ir}, \mathrm{Ni}, \mathrm{Pd}$ and $\mathrm{Pt}$, are magnetically ordered, exhibiting a gradual growth of their transition temperatures with increasing U-U distances [1]. Furthermore, magnetization measurements performed for all this series, except for UFeGe, in high magnetic fields up to $38 \mathrm{~T}$ at $4.2 \mathrm{~K}$, confirmed the paramagnetic properties of UCoGe and URuGe [2]. This systematics is apparently connected with the expected tendencies to yield an increase in the 5f-ligand hybridization (besides the $5 \mathrm{f}-5 \mathrm{f}$ overlap favored by small U-U separations) with decreasing the U-U distances gradually delocalizing the 5 f-electrons and in consequence leading to destabilizing the uranium magnetic moments. For example, by applying high magnetic fields at low temperatures in the case of UCoGe one first observes a linear behavior between 5 and $10 \mathrm{~T}$ and then a slight convex curvature of the magnetization with a field induced magnetic moment as large as $0.58 \mu_{\mathrm{B}}$ at $35 \mathrm{~T}[2,4]$. This indicates quite obviously that UCoGe is probably close to a ferromagnetic (FM) order, i.e., it might reveal the proximity to a quantum critical point (QCP). Precisely on such a border of FM order one can expect in this compound superconducting (SC) behavior mediated by critical magnetic fluctuations with the possible pairing of electrons into a spin triplet state [5]. Previously for UCoGe the absence of a magnetic order down to $1.2 \mathrm{~K}$ was reported, and an appreciable value of the linear specific-heat coefficient $\gamma(0 \mathrm{~K})$ of $65 \mathrm{~mJ} \mathrm{~K} \mathrm{~K}^{-2}$ $\mathrm{mol}^{-1} \mathrm{U}$ was established in UCoGe through specific heat measurements [4]. The electronic structure of these materials-being strongly depending on the occupation of the d-electron shell-indicates that the narrow 5f-band is located near or at the Fermi level, $\mathrm{E}_{\mathrm{F}}$, as is visible in recently published brief reports on possible weak ferromagnetism in UCoGe in Refs. [6], [7] and [8] containing band structure calculation results based on the full relativistic full potential local-orbital (FPLO) 
minimum basis code [9] within the local (spin)-density approximation (L(S)DA), used in $[6,8]$, and the WIEN2k package within $\mathrm{L}(\mathrm{S}) \mathrm{DA}[6]$ as well as $\mathrm{LSDA}+U$ [7] methods.

Recently a number of papers [10-18] have indicated the co-existence of weak ferromagnetic $(\mathrm{FM})$ and $\mathrm{SC}$ states in UCoGe below the critical temperature, $\mathrm{T}_{\mathrm{S}}=0.5$ $-0.7 \mathrm{~K}$. The p-wave type of superconductivity and an axial SC state with nodes along the easy magnetization direction along the $c$ axis was postulated [10]. Very recently a weak itinerant FM order has been detected in zero-field muon-spin rotation ( $\mu \mathrm{SR})$ measurements [16]. As the authors of Ref. [16] declared, the $\mu$ SR study provides an unambiguous proof for ferromagnetism present in the whole sample volume of the above germanide confirming the conclusions of earlier papers about the coexistence of ferromagnetism with superconductivity on the microscopic scale. Ferromagnetic quantum critical fluctuations (FM-QCF) and anomalous coexistence of ferromagnetism and superconductivity in UCoGe have been revealed also by CoNMR and NQR studies on a powder sample [17]. As a source of superconductivity in UCoGe the proximity of the system to the FM-QCP has been suggested. Interestingly, a high-pressure study of a single-crystalline UCoGe has recently revealed [18] that ferromagnetism is smoothly depressed and near the FM critical point superconductivity is enhanced to a maximum $\left(\mathrm{T}_{\mathrm{s}}=0.60 \mathrm{~K}\right)$ at $1.11 \mathrm{GPa}$ and is still present in the paramagnetic phase at pressures even above $2 \mathrm{GPa}$. Because of a limitation to only low temperature studies of all cited works [10-18] published on the above germanide up to now, we report here a set of new observations of the magnetic, transport and thermal properties in most cases for the first time given in a wide temperature range, measured also on a single-crystalline sample of UCoGe. 


\section{Experiment and calculations}

The single crystal having a shape of a cylinder of dimensions $l=5.0 \mathrm{~mm} \times \phi=1.5$ mm was grown by the Czochralski technique at the University of Silesia. The starting elements (with purity in weight percent) were: U (99.98), Co (99.99), and Ge (99.999). The quality of the single crystal before annealing (called as grown sample) was not high as indicates its temperature dependence of the resistivity (see below). The situation was completely changed after annealing the as grown sample at $900 \mathrm{~K}$ for 7 days in a high vacuum.

Magnetization and magnetic susceptibility measurements have been performed using a commercial superconducting quantum interference device (SQUID) magnetometer in the temperature region from $1.72 \mathrm{~K}$ up to $400 \mathrm{~K}$ and in fields up to $5 \mathrm{~T}$. The electrical resistivity measurements were performed using a steady-current four-point method with spot-welded wire contacts on cylindricallyshaped specimen at temperatures between $0.3 \mathrm{~K}$ and $300 \mathrm{~K}$ and in transverse magnetic fields $(\mathbf{j} \perp \mathbf{B})$ up to $\mathrm{B}=\mu_{0} \mathrm{H}=8 \mathrm{~T}$. The geometrical factor of the sample was determined with accuracy not lower than $20 \%$. The thermoelectric power in the low temperature range $(0.4-50 \mathrm{~K})$ and at the higher temperatures from $40 \mathrm{~K}$ to $360 \mathrm{~K}$ has been measured as described in Refs. [19,20], respectively. For thermoelectric power examinations the specimens were cut out to the dimensions of about $2.5 \times 1 \times 1 \mathrm{~mm}^{3}$ along the cylinder axis, being the [001] direction of the crystal. The temperature difference $(\Delta \mathrm{T})$ at lowest $\mathrm{T}$ was kept of the order $0.3 \mathrm{~K}$ along the sample while above $20 \mathrm{~K} \Delta \mathrm{T} \cong 2 \mathrm{~K}$. The thermal conduction was probed using the stationary heat flux method in the temperature range $5-300 \mathrm{~K}$. The difference $\Delta \mathrm{T}$ along the sample was 
in the range $0.1-0.5 \mathrm{~K}$. Particular care was taken to avoid a parasitic heat transfer between the sample and its environment. Specific heat measurements in the temperature range from 0.35 to $180 \mathrm{~K}$ were performed in a commercial multi-purpose measurement device (Quantum Design PPMS) with a $2 \tau$-relaxation-type method.

For the purpose to estimate diffusion term of thermoelectric power, we used the densities of states (DOS) of UCoGe that has been reported and discussed in [8]. They were calculated by employing the fully relativistic version of the FPLO method [9] in which the 4-component Kohn-Sham-Dirac equation, containing implicitly spinorbit coupling up to all orders, is solved self-consistently. The Perdew-Wang parametrization [21] of the exchange-correlation potential in the LDA approach was utilized. In the calculations the experimental values at room temperature of lattice parameters and atomic positions in the unit cell were used from tables 1 and 2 in [8]. The adopted valence basis sets were as follows: the U: 5d5f;6s6p6d;7s7p, Co: 3s3p3d;4s4p and Ge: 3s3p3d;4s4p states. The size of the k-point mesh of $12 \times 12 \times 12$ in the Brillouin zone turned out to be sufficient.

\section{Results and discussions}

\subsection{Magnetic properties}

The susceptibility data of UCoGe were measured in a field of $0.5 \mathrm{~T}$ along the cylindrical axis of the sample and perpendicular to it, which are approximately equivalent to the directions parallel, and perpendicular, respectively, to the crystallographic $c$ axis $\chi_{\mathbf{i}}$ (i means $\mathbf{B} \| c$ and $\mathbf{B} \perp c$ ). Figure 1(a) shows the temperature change of magnetic susceptibilities measured in these two directions for both kinds of samples, i.e., as grown and annealed ones. As seen from this figure, the differences 
between the $\chi_{\mathrm{i}}(\mathrm{T})$ curves obtained for the same sample but after different thermal treatment are negligible in the whole temperature range of measurements (shown are only data determined up to $100 \mathrm{~K}$ ). It means that these various treatments of the sample do not influence so much its magnetic behavior. Note, however, an existing pronounced anisotropy in the magnetic behavior between these two orientations of a single crystal. No sign of the susceptibility saturation in approaching $\mathrm{T}=0 \mathrm{~K}$ is apparent. In the inset of this figure, we have presented the field dependence of the magnetization $\mathbf{M}$ (in $\mu_{\mathrm{B}}$ ) in applied fields $\mathbf{B} \|_{\mathrm{c}}$ up to $5 \mathrm{~T}$ taken at various temperatures from 1.72 to $25 \mathrm{~K}$. One can see pronounced curvatures of this dependence diminishing with increasing temperature up to $25 \mathrm{~K}$, but without remanence $\mathrm{M}_{0}$ at each temperature measured. The lack of the field dependence of the susceptibility appears only above this temperature giving rise to a normal paramagnetic behavior. So that the FM correlations in UCoGe start to develop below $20 \mathrm{~K}$, which is well evidenced from the inset of figure 1(a). This is a clear sign of an existence of only strong magnetic correlations in this annealed sample over this temperature range. The induced magnetic moment at $1.72 \mathrm{~K}$ in a field of $5 \mathrm{~T}$ is equal to $0.14 \mu_{\mathrm{B}}$. A very similar magnetization dependence at the same temperature region (not shown) is noted also for $\mathbf{B} \perp c$ but with lower induced moments at $5 \mathrm{~T}$. For purpose of a comparison, we have also plotted in this inset the magnetization curve measured by Huy et al. [9] (solid line) on a single crystal of UCoGe at $2 \mathrm{~K}$ oriented along the $c$ axis. In contrast to our results, this curve displays the ferromagnetic behavior due to the presence of a spontaneous magnetization $M_{s}$ with a value of $0.07 \mu_{B}$. The total (intrinsic and induced) moment at $5 \mathrm{~T}$ was found to be about $0.21 \mu_{\mathrm{B}}$. After subtracting the value of their $\mathrm{M}_{\mathrm{s}}$ one obtains the same value of the induced moment 
found in our work. In order to better emphasize the behavior of the magnetization $\mathrm{M}$, in figure $1(\mathrm{~b})$ we have plotted the Arrott diagram (i.e. $\mu_{0} \mathrm{H} / \mathrm{M}$ vs. $\mathrm{M}^{2}$ ) at fixed temperatures from $1.72 \mathrm{~K}$ up to $25 \mathrm{~K}$. The isotherms that intersect the $\mathrm{M}^{2}$ axis indicate usually the presence of a spontaneous magnetization. In our case such an intersect is negligible and unusual only at $1.72 \mathrm{~K}$. A typical Arrott diagram should show linear isotherms, also that intersecting the origin of the coordinate system, which in the case of a ferromagnet indicates the $T_{C}$ temperature, as it was shown for the polycrystalline samples in Refs. [10] and [11]. As seen in figure 1(b), the above dependence measured at 1.72 and $5 \mathrm{~K}$ in higher values of $\mu_{0} \mathrm{H} / \mathrm{M}$ are almost identical to each other, but in lower values of $\mu_{0} \mathrm{H} / \mathrm{M}$ both show a strong curvature due to a substantial magnetic correlation effect and any $\mathrm{T}_{\mathrm{C}}$ can not be detected.

The temperature dependences of the inverse molar susceptibility along the above directions are displayed in figure 2. The obtained $\chi^{-1} \mathrm{i}(\mathrm{T})$ curves showing pronounced curvatures in all the temperatures measured can be fitted in the temperature range $100-400 \mathrm{~K}$ to the modified Curie-Weiss (MCW) law (denoted by the dashed lines) with the parameters given in the figure. Below about $100 \mathrm{~K}$ the temperature derivatives of the $\chi^{-1}{ }_{i}(T)$ curve become gradually increasing compared to the fitted MCW curves and, thus, this function diverges from the MCW fit $\chi^{-1}(T)=\left[\chi_{0}+N_{A} \mu_{e f f}^{2} / 3 k_{B}\left(T-\Theta_{p}\right)\right]^{-1}$ where $\chi_{0}, \mathrm{~N}_{\mathrm{A}}, \mathrm{k}_{\mathrm{B}} . \mu_{\mathrm{eff}}$, and $\Theta_{\mathrm{p}}$ have usual meaning. From the point of view of the crystal electric field (CEF) theory such a rapid $\chi^{-1}{ }_{i}$ vs. T bending can be easily explained by thermal depopulation of CEF levels with decreasing temperature. In the case of the $5 \mathrm{f}^{2}$ configuration of uranium atom one should expect rather a saturation tendency of these curves to a constant value at low temperatures (Van-Vleck paramagnetism) due to the low symmetry of the 
orthorhombic crystal structure of UCoGe yielding only CEF singlets. The observed curvature could be, however, well explained by the $5 f^{3}$ uranium electronic configuration for which in this symmetry Kramer's doublets arise. However, one can not exclude the influence of the paramagnetism of Co atoms. As some paramagnetic data for several ternary uranium compounds containing cobalt indicate (see for example cases of $\mathrm{UCoAl}$ and its solid solutions with $\mathrm{Ni}[22,23]$ and $\mathrm{U}_{2} \mathrm{CoSi}_{3}$ [24]) a strong curvature of their $\chi_{\mathrm{i}}^{-1}(\mathrm{~T})$ takes place. It should be mentioned that the obtained high temperature results are in reasonable accordance with the previous data published in Ref. [1]. There is, however, some difference in the effective magnetic moments between our present value $\mu_{\mathrm{eff}}=1.98(5) \mu_{\mathrm{B}}$ (this paper) and that given in Ref. [12] of 1.6(1) $\mu_{B}$ (no curve is shown). A similar curvilinear behavior of $\chi^{-1}{ }_{i}(T)$ has been reported for the solid solutions of the polycrystalline $\mathrm{U}\left(\mathrm{Co}_{1-\mathrm{x}} \mathrm{Ru}_{\mathrm{x}}\right) \mathrm{Ge}$ samples for $\mathrm{x}=0.4-1.0$ (see Ref. [11]) measured in a field $\mathrm{B}=1 \mathrm{~T}$ in the temperature range $2-300 \mathrm{~K}$. In the upper inset of figure 2 we show the temperature variation of the magnetization $\sigma_{\mathrm{i}}(\mathrm{T})$ taken at $0.5 \mathrm{~T}$ while in the lower inset we display a field dependence of the magnetization $\mathrm{M}_{\mathrm{i}}(\mathrm{B})$ measured only at $1.72 \mathrm{~K}$. As seen, in our case the curvilinear decrease in $\mathrm{M}_{\mathrm{a}}(\mathrm{B}) \mathrm{o} \%$ served down to $0 \mathrm{~K}$ for both orientations of the sample is without hysteresis, i.e., no magnetic remanence exists. Therefore, it can be related only to the presence of ferromagnetic correlations (FMC) in contrary to having the spontaneous moment observed earlier on both the polycrystalline [10,12] $\left(0.029 \mu_{\mathrm{B}}\right)$ and single-crystalline [13] $\left(0.07 \mu_{\mathrm{B}}\right)$ samples. The upper inset of figure 2 reveals only a negligible change in a slope of these two curves at the temperature expected for the ferromagnetic transition $\mathrm{T}_{\mathrm{C}}=3 \mathrm{~K}$. 


\subsection{Transport properties}

\subsubsection{Resistivity and magnetoresistivity}

The electrical resistivities measured previously by different authors on the polycrystalline and single-crystalline samples of UCoGe have been presented so far only in the small ranges of low temperatures, namely between $0.02-8 \mathrm{~K}$ [10], $0.23-$ $10 \mathrm{~K}[12,13], 0.4-8 \mathrm{~K}$ [14] and 0.25 - 10 [15] Contrastingly, we presents in figure 3 the temperature dependences of the resistivity measured in a wide temperature range, i.e., between 0.3 and $300 \mathrm{~K}$ for both kinds of UCoGe samples.

As is apparent in figure 3, the quality of our single crystal before annealing was not so high. The transport measurement data show rather a poor residual resistivity ratio RRR of about 2 . It seems that this was mainly caused by a small deviation of the $c$ axis of crystallites from the cylinder axis yielding an additional electron scattering on the crystallite separations. It appears, however, that the quality of the same single crystal, but annealed at $900 \mathrm{~K}$ for one week, has been distinctly improved by reaching the RRR ratio of 19 . The resistivity data of this annealed sample are also plotted against temperature in this figure. Nevertheless, despite the difference in the absolute magnitudes of the resistivity, especially at low temperatures, and the lack of the superconductivity down to $0.3 \mathrm{~K}$ for the nonannealed sample, the temperature characteristics of the resistivity for both samples have almost identical features, as shown in the insets to figure 3 . In contrast to the previously published data reported by other authors, both these samples exhibit at low temperatures the linear $\rho(T)$ dependence and, in addition, the inflection point $T_{\text {inf }}$ at about $13 \mathrm{~K}$ with further linear behavior when the temperature is increased above this 
point (see the left hand inset of figure 3). An analogous electrical behavior measured up to $25 \mathrm{~K}$ on polycrystalline $\mathrm{UCoGe}$ has recently been reported by Huy and de Visser [25]. In turn, in the right hand inset of figure 3 the temperature derivative of the resistivity is plotted against the temperature. For both samples a wide maximum around $\mathrm{T}_{\mathrm{inf}}$ is well apparent underlining strongly the fact that at this temperature there is a crossover probably dividing the energy scale for the here-studied compound into low-temperature and higher temperature regions. The aforementioned linearity is better seen on an enlarged picture of the low-temperature curve of $\rho(T)$ presented in figure 4, where also no sign of a distinct anomaly around $3 \mathrm{~K}$ is observed, i.e., at the temperature claimed as a Curie point for the polycrystalline and single-crystalline samples studied by other authors (see Refs. [10-18]). Recently, such a linear behavior above the corresponding $\mathrm{T}_{\mathrm{sc}}$ 's has been found in the super clean samples of $\mathrm{URu}_{2} \mathrm{Si}_{2}$ [26] and $\mathrm{NpPd}_{5} \mathrm{Al}_{2}$ [27]. Also for the $\mathrm{URh}_{0.62} \mathrm{Ru}_{0.38} \mathrm{Ge}$ solid solution lying on the border of ferromagnetism the non-Fermi liquid (NFL) regime has been reported [28]. Thus, in the latter $\mathrm{U}(\mathrm{Rh}, \mathrm{Ru}) \mathrm{Ge}$ system the randomness interactions between localized and itinerant electrons acting near the critical concentration $\mathrm{x}_{\mathrm{cr}} \approx 0.38$ are thought to be an origin of a NFL behavior (see e.g. Ref. [29]), supported also by the lowtemperature $\mathrm{C}_{\mathrm{p}} \sim \mathrm{T} \ln \mathrm{T}$ dependence [30].

On the contrary, the $\mathrm{T}^{2}$ Fermi liquid law has been reported in all cases mentioned above [10-14, 25]. In Ref. [15] The $\mathrm{T}^{2}$ dependence is reported below $\mathrm{T}_{\mathrm{C}}$ and $\mathrm{T}^{5 / 3}$ above this temperature up to $10 \mathrm{~K}$. Since all these samples were weakly ferromagnetic at low temperatures, such a behavior was explained, e.g., in Ref. [12] by scattering on magnons with the same exponent. Figure 4 also clearly illustrates the $\mathrm{SC}$ transition at $\mathrm{T}_{\mathrm{SC}}=0.65 \mathrm{~K}$. Furthermore, in the next figure 5 we show the 
difference $\Delta \rho_{i}\left(\rho-\rho_{0}\right)$, where $\rho$ is a measured resistivity and $\rho_{0}$ is the residual one, plotted against temperature for the annealed sample of UCoGe $\left(\Delta \rho_{1}\right)$ and for reference sample of ThNiGe $\left(\Delta \rho_{2}\right)$. We have not used here as a reference, e.g., ThCoGe because the latter compound exhibits some weak ferromagnetic behavior up to about 200-250 $\mathrm{K}$, as we and the authors of Ref. [31] have found. The crystal structure of these two Th-based ternaries is, however, of the hexagonal $\mathrm{AlB}_{2}$-type and, therefore, both these ternaries are not isostructural with the orthorhombic TiNiSi-type of UCoGe, but at present no other possibility exist to use a better reference material.

As a result of using the non-magnetic reference we display in figure 5 the resulting curve for $\operatorname{UCoGe}\left(\Delta \rho_{1,2}=\Delta \rho_{1}-\Delta \rho_{2}\right)$ which distinctly shows the Kondo-like behavior above about $80 \mathrm{~K}$. As is also clear from this figure, as well as from figure 6, where we plotted the above difference in a manner $\Delta \rho_{1,2}$ vs. $\log \mathrm{T}$, the high temperature Kondo-like effect is preceded by a knee in these curves. The maximum as observed above, marked as $\mathrm{T}_{\mathrm{coh}}$, is usually associated with the coherence effect in the Kondo-lattice system [32]. The fitting parameters are also shown in this figure.

The measured overall temperature dependence of the electrical resistivity of UCoGe resembles that of other intermetallic uranium ternaries, but especially that of $\mathrm{URu}_{2} \mathrm{Si}_{2}$ [34]. In a similar way to the case of $\mathrm{URu}_{2} \mathrm{Si}_{2}$, our $\Delta \rho_{1,2}(\mathrm{~T})$ decreases also rapidly below $50 \mathrm{~K}$ down to a residual resistivity at its superconducting temperature. Previously, the only existing $\rho(\mathrm{T})$ curve, obtained for UCoGe in a wide temperature range $1.5-300 \mathrm{~K}$, has been reported by Canepa et al. [35]. Despite annealing of their polycrystalline sample (at $1250 \mathrm{~K}$ for 3-5 days), the overall resistivity values given by them are almost one order of magnitude larger than those of our annealed sample. Moreover, the latter authors observed some sharp maximum in their $\rho(\mathrm{T})$ at $\mathrm{T}_{\max }=46$ 
$\mathrm{K}$ instead of our smooth "knee", which they interpreted as an evidence of nonidentified magnetic transition at this temperature.

Additionally, in the inset to figure 6 we have plotted the temperature dependence of the resistivity for $\mathrm{ThNiGe}$ with fitting these experimental results into the modified Bloch-Grüneisen-Mott equation [33] allowing to determine the Debye temperature $\Theta^{R}{ }_{D}(=139 \mathrm{~K})$ for this compound. Our overall results of the resistivity found for this Ni-based ternary material is by a factor of about 5 lower than those published in the literature [31].

Finally, in figures 7 (a) and 7 (b) the low temperature resistivity $\rho(T)$, measured in fields $\mathrm{B}=0$ and $8 \mathrm{~T}$ as well as transverse magnetoresistivity (TMR), defined as $\Delta \rho / \rho=[\rho(B, T)-\rho(0, T)] / \rho(0, T) \times 100(\%)$ are shown, respectively. If for $\mathrm{B}=0$, as indicated above, this former dependence is linear in temperature, the situation is changed into the Fermi liquid (FL) behavior at $8 \mathrm{~T}$ (see the inset of figure 7(a)). So that the FL state has been recovered in applied magnetic fields. In figure 7(b), where we have plotted the $\Delta \rho / \rho(\mathrm{T})$ curve below $60 \mathrm{~K}$ measured at $8 \mathrm{~T}$, a strong negative increase in TMR with lowering $\mathrm{T}$ is apparent with an appearance of a sharp negative minimum at $8 \mathrm{~K}$ which reason is at present unknown. Furthermore, as the inset to this figure clearly shows, the characteristic temperature $T_{\text {inf }}$ is marked at about $13 \mathrm{~K}$, i.e. exactly at the same temperature as found from the temperature derivative of the resistivity as the crossover temperature described above.

Next, we show in figure 8 the TMR as a function of a field B (applied up to 8 T) taken at different temperatures $(4.2-40 \mathrm{~K})$. For all temperatures measured we have obtained the negative dependences of TMR, which character, however, changes with decreasing temperature. It appears that this is typical of the Kondo-lattice-like 
behavior (convex curve) at temperatures above $15 \mathrm{~K}$, but below this temperature it becomes an S-shaped one in the range of $15-10 \mathrm{~K}$ to become, finally, almost concave in fields at $4.2 \mathrm{~K}$ as is expected for a ferromagnet. At the latter temperature, $\Delta \rho / \rho(\mathrm{B})$ achieves the large negative overall value up to $8 \mathrm{~T}$, where it attains about -25 $\%$, however it still increases negatively up to $-28 \%$ reaching its maximum at about 8 $\mathrm{K}$. The character of field and temperature dependencies of the as grown sample of UCoGe was analogous, but the maximum value of TMR was found by a factor of 2.8 lower $(-10 \%)$. The increase in the absolute value of TMR with lowering the temperature, as is presented in figure 8 , indicates that down to almost $T_{\text {inf }}$ an expected Kondo-lattice-dependence takes place. A change in the observed overall slopes gives rises to the crossover around $13 \mathrm{~K}$ mentioned above. The dashed lines illustrate the $\alpha\left(\mu_{0} \mathrm{H}\right)^{2}$ dependencies with the $\alpha$ coefficient varied with temperature.

\subsubsection{Thermoelectric power}

The measured Seebeck coefficient $\mathrm{S}(\mathrm{T})$ of UCoGe is displayed in figure 9. As seen, the main feature of this curve is a pronounced positive maximum at $\mathrm{T}_{\max }=13 \mathrm{~K}$ which coincides well with the temperatures of maxima in the temperature derivatives of both the resistivity (right hand inset of figure 3) or magnetoresistiviy (insert of figure 7(b)) and also with $T_{\max }$ of the smaller peak in $\Delta \mathrm{C}_{\mathrm{p}}(\mathrm{T})$ (see below the inset of figure 12(b)). As we have pointed out in the previous sections of this paper, this temperature is in our system under study a measure of the characteristic crossover temperature, denoted

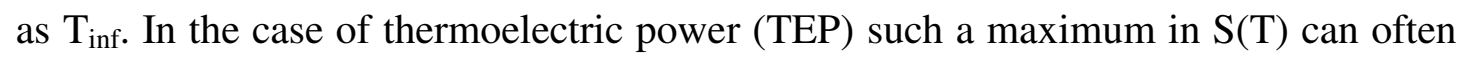
be interpreted as being due to the Kondo lattice effect [36], where $T_{\max }=T_{K} / 3$ or for very clean samples as a phonon drag effect [37]. However, the latter should take place 
at about $\Theta_{\mathrm{D}} / 10$, i.e., at about $25 \mathrm{~K}$ for $\Theta_{\mathrm{D}}=248 \mathrm{~K}$ being the Debye temperature derived in the low temperature region of the specific heat of UCoGe (see lower inset of figure 12(a)). On the other hand, the TEP of UCoGe resembles the behavior of superconducting, spin fluctuating $\mathrm{UPt}_{3}[38,39]$, where the positive peak at $\mathrm{T} \sim 8 \mathrm{~K}$ is considered as a measure of the spin fluctuation temperature $\mathrm{T}_{\text {sf }}$. It should be also mentioned here that a very similar TEP and resistivity behavior exhibits $\mathrm{U}_{3} \mathrm{Au}_{3} \mathrm{Sn}_{4}$ [40], which is considered as a heavy fermion system with $\gamma(0)=280 \mathrm{~mJ} \mathrm{~K}^{-2} \mathrm{~mol}^{-1} \mathrm{U}$. The large $\gamma$ value is predominantly caused by a reduced $\mathrm{f}$ - $\mathrm{d}$ hybridization.

Furthermore, according to the phenomenological model which considers scattering conduction electrons by the $5 \mathrm{f}$ quasi-particle band of a Lorentzian form, used in Ref. [41] at higher temperatures for the TEP results of correlated cerium and uranium systems, $S(T)$ can be described using the following equation: $S(T)=A_{i} T /\left(B_{i}{ }^{2}\right.$ $+\mathrm{T}^{2}$ ), where $\mathrm{i}=\mathrm{N}$ or $\mathrm{W}$ in the case of a narrow or wide band of the $5 \mathrm{f}$ electrons, respectively. From a fitting of this equation to the low temperature $S(T)$ results we can estimate roughly the f-electron level position to the Fermi level, $\Delta=\varepsilon_{\mathrm{f}}-\mathrm{E}_{\mathrm{F}}$, and the width $\Gamma$ of the band in a Lorentzian form, because $A_{N}=2 \Delta /$ lel and $B_{N}^{2}=3\left(\Delta^{2}+\right.$ $\left.\Gamma^{2}\right) /\left(\pi \mathrm{k}_{\mathrm{B}}\right)^{2}$. As shown in the lower inset of figure 9, the TEP peak in UCoGe can be well fitted by the narrow, single Lorentzian band (SLB) model, but only up to $30 \mathrm{~K}$. Above this temperature, the theoretical curve diverges upward from the experimental one. This deviation from the SLB model observed at higher temperatures may be connected with scattering processes that are beyond this model, like a phonon-drag effect (see below), crystal field interactions, and so on. For the parameters $A_{N}$ and $B_{N}$ given in the inset we find $\Delta=0.26 \mathrm{meV}$ and $\Gamma=2 \mathrm{meV}$. This might indicate that in UCoGe we have to do with more localized character of the 5f-electrons which, 
however, below the crossover temperature become more itinerant. The latter is also consistent with the linear behavior of $\mathrm{S}(\mathrm{T})$ characteristic of normal metals as is observed for UCoGe below about $2.5 \mathrm{~K}$ (upper inset of figure 9). According to the equation followed by metals: $\mathrm{S}(\mathrm{T})=\alpha \mathrm{T}$, where $\alpha=\pi^{2} \mathrm{k}_{\mathrm{B}} / 3$ lelT $\mathrm{T}^{*}$, in our case $\alpha \sim 2.8 \mu \mathrm{V}$ $\mathrm{K}^{-2}$ which yields $\mathrm{T}^{*} \sim 100 \mathrm{~K}$, just as the value $\mathrm{T}_{\mathrm{K}}$, determined from the Kondo-like peak in the specific heat (see figure 12(b) and the corresponding text). The value $\alpha$ is practically the same for both kinds of samples used in our studies, so that we present in figure 9 only the results obtained on the as grown sample. The difference between TEPs in these two samples, except for a small difference in their magnitudes, is well seen only below about $0.8 \mathrm{~K}$ where the annealed sample starts to go into the SC state, where TEP drops to 0 .

In order to estimate an overall magnitude of the temperature dependence of TEP in UCoGe, we have applied the Mott model for diffusion TEP, $S_{d}(T)$, of a twoband conductor [42] in an analogous manner as it was done for UCoGas in Ref. [43] using the following formula:

$$
\mathrm{S}_{\mathrm{d}}(\mathrm{T})=\frac{\pi^{2} \mathrm{k}_{\mathrm{B}}^{2} \mathrm{~T}}{3 \mathrm{e}}\left\{\left[\frac{1}{\mathrm{~N}_{s}(\mathrm{E})} \frac{\mathrm{dN}_{s}(\mathrm{E})}{\mathrm{dE}}\right]_{\mathrm{E}=\mathrm{E}_{\mathrm{F}}}-\left[\frac{1}{\mathrm{~N}_{p d f}(\mathrm{E})} \frac{\mathrm{dN}_{p d f}(\mathrm{E})}{\mathrm{dE}}\right]_{\mathrm{E}=\mathrm{E}_{\mathrm{F}}}\right\},
$$

where $\mathrm{N}_{s}$ and $\mathrm{N}_{p d f}$ denote the sums of contributions to densities of states coming from all conduction electrons, i.e $s$ and $p, d, f$ ones, respectively. We used our calculated, employing the FPLO code [9] in the LDA approach, $\mathrm{N}_{s}$ and $\mathrm{N}_{p d f}$ densities in paramagnetic UCoGe, reported in [8], and computed their derivatives over energy at $\mathrm{E}_{\mathrm{F}}$, being presented in figure 10. As is seen in this figure, the $\mathrm{N}_{s}$ contribution in UCoGe is equal to 0 (see triangles), thus also its derivative. Hence, only the second term connected with the $\mathrm{N}_{p d f}$ density remains non-zero in the above equation. In this 
way, we obtained the linear dependence of diffusion TEP as follows: $S_{d}(T)=-$ $14.7 \times 10^{-2} \mathrm{~T}\left(\mu \mathrm{V} \mathrm{K}^{-1}\right)$, which is plotted in figure 9 (dashed straight line). It is seen that the calculated $S_{d}(T)$ yields a negative linear temperature variation reflecting the general trend of the experimental TEP at higher temperatures.

It is interesting to add that in the case of the strong scattering of phonons by electrons, which has been noted in connection with the thermal conductivity of UCoGe considered below, the TEP comprises mainly diffusion $S_{d}$ and a phonon drag $\mathrm{S}_{\mathrm{ph}}$ contributions. If one takes the former one, $\mathrm{S}_{\mathrm{d}}(\mathrm{T})$, being negative in the whole temperature region studied, just as that found from the above calculations, then the latter, $S_{\mathrm{ph}}(T)$, should have only positive values, and their sum $S_{d}(T)+S_{p h}(T)$, would give the resulting shape of our measured TEP depicted in figure 9. Such an approach, e.g., has been proposed for the superconducting cuprates [44] and could also be applied in our case, but it requires at least to perform neutron inelastic scattering measurements on a large single crystal of UCoGe. It turned out, that for several uranium compounds, like $\mathrm{UNi}_{4} \mathrm{~B}, \mathrm{UPt}_{2} \mathrm{In}$, and $\mathrm{UCu}_{3+\mathrm{x}} \mathrm{Ga}_{2-\mathrm{x}}$ as well as for $\mathrm{UNi}_{2} \mathrm{Al}_{3}$ and $\mathrm{UPd}_{2} \mathrm{Al}_{3}$, two 5 f-band Mott's Lorentzian model, where both $\mathrm{i}=\mathrm{N}$ and $\mathrm{W}$ cases had to be used together. Thus, this signals the presence of two energy scales in these systems. It is common, however, for all these materials that their TEP increases with an upward curvature, i.e., in an opposite way to the TEP of UCoGe, and with a tendency to saturate in the vicinity of RT. Nevertheless, calculations taking only $\mathrm{i}=$ $\mathrm{N}$, yield both the TEP-curve shape and the above parameters (see Table 2 in Ref. [45 ]) close to ours.

Another feature of the here-described $\mathrm{S}(\mathrm{T})$ is a change of the sign from positive to negative at about $75 \mathrm{~K}$, which is easy to explain in the case described 
above, i.e., due to the sum of negative $S_{d}(T)$ and positive $S_{p h}(T)$ contributions. Apart from this description, the positive values of $\mathrm{S}$ found in the lower temperature region may indicate that the principal charge carriers are holes. It also means that the lack of charge compensation by the transfer of conduction electrons from uranium to Co and Ge atoms creates holes, and only in the high temperature region the electron-type conductivity starts to dominate. The large value of $\mathrm{S}$ of about $20 \mu \mathrm{V} \mathrm{K} \mathrm{K}^{-1}$ observed for UCoGe at $\mathrm{T}_{\max } \equiv \mathrm{T}_{\mathrm{inf}}$ is attributed to Kondo-lattice type of interactions also reflected by fairly large $\mathrm{C}_{\mathrm{p}} / \mathrm{T}$ values in this region of temperature (see below). At higher temperatures $S(T)$ has a tendency to saturate into a large negative value of $-30 \mu \mathrm{V} \mathrm{K}^{-1}$.

\subsubsection{Thermal conductivity}

Thermal conductivity $\kappa_{\mathrm{tot}}(\mathrm{T})$ data obtained on the annealed sample of UCoGe, taken over a range $5-300 \mathrm{~K}$, are given in figure 11 . This shows that $\kappa_{\text {tot }}$ first increases very steeply with rising temperature, then in the vicinity of $25 \mathrm{~K}$ it goes through a wide knee and finally increases monotonically to RT but having a small anomaly of unknown origin around $200 \mathrm{~K}$. At low temperatures up to about $15 \mathrm{~K}$, this dependence can be approximated as a linear one. The total thermal conductivity of a non-magnetically ordered material as UCoGe is usually expressed as a sum of the electronic $\kappa_{\mathrm{e}}$ and lattice $\kappa_{\text {lat }}$ contributions. At first, we have estimated the contribution of the electron thermal conductivity to the total thermal conductivity by using the Wiedemann-Franz (W-F) law: $\kappa_{\mathrm{e}}=\mathrm{L}_{0} \mathrm{~T} / \rho$, where $\mathrm{L}_{0}$ is the So mmerfeld value $2.45 \times 10^{-}$ ${ }^{8} \mathrm{~W} \Omega \mathrm{K}^{-2}$. As seen from this figure, $\kappa_{\mathrm{e}}$ is essentially a linear function of temperature but having only about $15 \%$ value of $\kappa_{\text {tot }}$ at RT. Such a small magnitude of the electronic part of the thermal conductivity is caused by large overall values of the 
electrical resistivity (see figure 3) being far from that exhibited by typical metallic systems. The steep decrease of the resistivity below $50 \mathrm{~K}$, owing to the coherence effect, is here connected with an almost linear drop of $\kappa_{\text {tot }}(T)$ at the same temperature region. A calculation of the Wiedemann-Franz-Lorenz number $L=\kappa_{\text {tot }} \rho / T$ using the measured thermal conductivity gives abnormal high values for $\mathrm{L}(\mathrm{T})$ with a clear evidence that a large lattice term $\kappa_{\text {lat }}$ exists in the total thermal conductivity of UCoGe, several times larger than that of the electronic $\kappa_{\mathrm{e}}$ one. The temperature dependence of the normalized Lorenz number, $\mathrm{L}_{\mathrm{L}}$, is given in the inset of figure 11 . This dependence goes through a well apparent maximum at $\mathrm{T}_{\max } \sim 25 \mathrm{~K}$ where this ratio reaches a value of about 18 and then it monotonically declines with further increasing temperature to reach finally at RT a value being 7 times higher than $\mathrm{L}_{0}$ itself. Such a large value at higher temperatures is often an obstacle to observe a Kondo-like behavior in the thermal conductivity [46]. This is possible, however, e.g. for $\mathrm{CeCu}_{2}$ and $\mathrm{YCu}_{2}$, also crystallizing in an orthorhombic system like UCoGe, which however show up $\mathrm{L} \sim \mathrm{L}_{0}$ [47]. On the other hand, the overall shape of the $\mathrm{L} / \mathrm{L}_{0}(\mathrm{~T})$ function found for $\mathrm{UCoGe}$ is very similar to those, e.g. of $\mathrm{UPd}_{2} \mathrm{Al}_{3}$ [48] or $\mathrm{CeCu}_{2} \mathrm{Si}_{2}$ [49] and other heavy fermion superconductors. Figure 11 shows that separation of the lattice contribution trough $\kappa_{\text {lat }}=\kappa_{\text {tot }}-\kappa_{\mathrm{e}}$ yielded the temperature dependence $\kappa_{\text {lat }}(\mathrm{T})$ close to that of $\kappa_{\text {tot }}(T)$. Thus, the large Lorenz ratio is due to unusual large ratio $\kappa_{\text {lat }} / \kappa_{\mathrm{e}}$. Furthermore, the lattice component is generally assumed to be composed of several other contributions, like phonon, non-magnetic bipolaron and excitonic ones, depending on the kind of heat carriers [50,51]. Then, the phonon conductivity, $\kappa_{\mathrm{ph}}$ may be described by the Debye model given by Callaway's equation [52] as described in details in Refs. [53] and [54]. 


$$
\kappa_{\mathrm{ph}}=\mathrm{GT}^{3} \int_{0}^{\Theta / T} \frac{x^{4}}{\tau^{-1} \sinh ^{2}(x / 2)} d x
$$

where $\mathrm{x}=\hbar \omega / \mathrm{k}_{\mathrm{B}} \mathrm{T}$ ( $\hbar \omega$ is the phonon energy), $\mathrm{G}$ is a constant, $\Theta_{\mathrm{D}}$ is the Debye temperature, and $\tau^{-1}$ is the sum of inverse relaxation times for various phonon scattering processes $[53,54]$. In the calculations we have used two parameters, i.e., sound velocity $v_{p h}=900 \mathrm{~m} / \mathrm{s}$ and Debye temperature $\Theta_{\mathrm{D}}=250 \mathrm{~K}$. For the other contributions, such as the bipolar ( $\left.\kappa_{\text {bip }}\right)$ and excitonic $\left(\kappa_{\text {exc }}\right)$ ones, the heat transport is well described in a review given by Smirnov and Oskotski in Ref. [50].

Figure 11 presents the separation of $\kappa_{\text {lat }}($ exp. $)$ into $\kappa_{\mathrm{ph}}$ (calc.) and a difference $\Delta \kappa_{\text {lat }}$. It is clearly seen that Calleway's equation well describes the phonon contributions, $\kappa_{\mathrm{ph}}$ (calc.), to $\kappa_{\text {tot }}$ at low temperatures, but above $25 \mathrm{~K}$ it diverges from $\kappa_{\text {lat }}($ exp.) and diminishes with increasing temperature to reach a value at $\mathrm{RT}$ as that of $\kappa_{\mathrm{e}}$ one. The remaining part after the subtraction of $\kappa_{\mathrm{ph}}(\mathrm{calc}).(\mathrm{T})$ from $\kappa_{\mathrm{lat}}(\mathrm{exp}).(\mathrm{T})$, i.e., the difference $\Delta \kappa_{\text {lat }}$, we have assumed to be mainly due to $\kappa_{\text {bip }}(T)$ in such a semimetallic compound as UCoGe. This differential function starting from $25 \mathrm{~K}$ follows a shape almost that of high temperature $\kappa_{\text {tot }}(T)$. As we have pointed out in the paper [53], the Callaway equation, however, omits several other possible phonons scattering contributions, e.g., the scattering on the moments in the PM state [50] and hence the difference $\Delta \kappa_{\text {lat }}$ may consist (except for bipolarons) also of other different but difficult to be identified contributions. If we have actually to do with bipolarons in the compound under consideration, a question arises about their role in the occurrence of SC in UCoGe.

\subsection{Thermal properties}


We show in figure 12(a) the temperature dependences of the specific heat $C_{p}$ measured up to $180 \mathrm{~K}$ for both UCoGe (non-annealed) and ThNiGe; the latter is taken as a non-magnetic reference of the phonon contribution to the total $\mathrm{C}_{\mathrm{p}}$, as it has been done in the case of the transport properties. At lower temperatures, the specific heat over temperature which can be fitted by the equation: $\mathrm{C}_{\mathrm{p}} / \mathrm{T}=\gamma(0)+\mathrm{BT}^{2}$, where the first and second terms are electronic and phonon contributions, respectively, is shown in the lower inset. The linear response of the above function is apparent for both studied compounds up to $7 \mathrm{~K}$. The values of the electronic specific heat coefficient $\gamma(0)$ and the Debye temperature $\Theta_{\mathrm{D}}$ obtained for these two compounds are displayed in this inset. Our $\gamma(0)$ values being of 54 and $7 \mathrm{~mJ} \mathrm{~K}^{-2} \mathrm{~mol}^{-1}$ are close to the literature values of 57 [13] and 5 [55] or $7.6 \mathrm{~mJ} \mathrm{~K}^{-2} \mathrm{~mol}^{-1}$ [31], respectively. The enhancement of the $\gamma(0)$ value by the existence of the 5 f-electrons is here almost 8 times. Nevertheless, these values given for UCoGe are not so large compared to those of real heavy-fermion systems (more than $400 \mathrm{~mJ} \mathrm{~K}^{-2} \mathrm{~mol}^{-1}$ ). On the other hand, the Debye temperature of UCoGe is, as expected, somewhat smaller than that for ThNiGe, i.e., 248 against $266 \mathrm{~K}$. The latter value is comparable to $255 \mathrm{~K}$ found in Ref. [31] and to $246 \mathrm{~K}$ reported in Ref. [55]. Despite different crystal structures of these two compounds, the closeness in their Debye temperatures strongly indicates a similarity in their lattice dynamics and justifies using here $\mathrm{ThNiGe}$ as a reference non-magnetic compound. The upper inset illustrates the $C_{p} / T$ vs. $T$ dependences for our two kinds of samples of UCoGe compared to that reported by Huy et al. [13] but in contrast to our results showing the ferromagnetic properties with $\mathrm{T}_{\mathrm{C}} \sim 3 \mathrm{~K}$. It is surprising that our annealed sample, though having no sign of FM order, shows a similar in shape anomaly but being more enhanced as to its magnitude. Certainly both of them do not 
reveal a $\lambda$-type-shape anomaly as expected in the case of the magnetic order. It should be noted that our as grown sample although exhibits the low-temperature linear behavior in $\rho(T)$, it gives a completely smooth trend in the $C_{p} / T$ against $T$ curve which allowed for a simple extrapolation to $\mathrm{T}=0 \mathrm{~K}$ to get the $\gamma(0)$ value for UCoGe. On the other hand, the revealed large hump existing in our low temperature specific heat measurements in the annealed sample of UCoGe (see the upper inset of figure 12 (a)) makes it difficult to judge about any divergent tendency of the $C_{p} / T$ vs. $\log T$ function expected for the NFL state, which is well manifested by the observed linearity in $\rho(T)$ described above. As seen from this inset, all three considered there cases approach the same $C_{p} / T$ values above $5 \mathrm{~K}$. In addition, there is also shown a smooth $\mathrm{C}_{\mathrm{ph}}(\mathrm{T})$ curve defined as a sum of the Debye and Einstein contributions to $\mathrm{C}_{\mathrm{p}}$ of ThNiGe, as specified below. This procedure allows to eliminate $\mathrm{C}_{\mathrm{el}}$ of a given Thcompound and signals that $\mathrm{C}_{\mathrm{ph}}$ is more complex than usual when one uses only the Debye function. Thus, in figure 12(b) we display the specific heat difference $\Delta C_{p}=$ $\mathrm{C}_{\mathrm{p}}-\mathrm{C}_{\mathrm{ph}}$ plotted against $\mathrm{T}$. We found the difference $\Delta \mathrm{C}_{\mathrm{p}}$ between these two curves of large numbers, being magnetic and electronic in origin. An expected error in $\Delta C_{p}$ is usually a sum of errors made during measurements of both heat capacities. To minimize the experimental uncertainties in an extraction $\Delta \mathrm{C}_{\mathrm{p}}$, we have used as $\mathrm{C}_{\mathrm{ph}}(\mathrm{T})$, i.e. a smooth curve mentioned above, which is without $\mathrm{C}_{\mathrm{el}}$ of $\mathrm{ThNiGe}$ (see below).

As Figure 12(b) indicates, as a result of the above procedure one gets a "camel curve", i.e. there are possible two wide anomalies spanned in the temperature range $1.0-90 \mathrm{~K}$. Their maxima are centered at about 13 and $45 \mathrm{~K}$ and their amplitudes are in a rough ratio of about 1:2, respectively. In addition, we present at 
these temperatures the presumably bar errors estimated as \pm 5 and $\pm 2 \%$, respectively. Similar error values have been used by Rietschel et al. [57] in their f-derived $\Delta \mathrm{C}_{\mathrm{p}}$ for a number of magnetically non-ordered uranium and rare-earth compounds as $\mathrm{UBe}_{13}$, $\mathrm{UPt}_{3}, \mathrm{CeCu}_{2} \mathrm{Si}_{2}$ and $\mathrm{CeCu}_{6}$ showing superconductivity at very low temperatures, where they considered the superposition of Kondo and Schottky peaks in these compounds, having also to do with subtracting from each other the large numbers.

This is well seen in the inset to this figure when subtracting the overall electronic specific heat $\mathrm{C}_{\mathrm{el}}{ }^{*}=\gamma^{*} \mathrm{~T}$ from $\Delta \mathrm{C}_{\mathrm{p}}(\mathrm{T})$ of $\mathrm{UCoGe}$, but with a gamma value $\gamma^{*}$ amounting only $\sim 1 / 3 \gamma(0)$, as presented in figure 12(b). Of course, the temperature change in a gamma value from $\gamma(0)=54.0 \mathrm{~mJ} \mathrm{~K}^{-2} \mathrm{molU}^{-1}$ found at $\mathrm{T}=0 \mathrm{~K}$ to a constant value $\gamma^{*}=18.5 \mathrm{~mJ} \mathrm{~K}^{-2} \mathrm{molU}^{-1}$, valid above $90 \mathrm{~K}$, is unknown. However the "camel curve" as well as the straight-line $\mathrm{C}_{\mathrm{el}}{ }^{*}=\gamma^{*} \mathrm{~T}$ are well followed by experimental points, which may indicate a good extracting procedure applied and that the latter can not be treated as any Schottky contribution. The occurrence of the $13 \mathrm{~K}-$ anomaly seems to be associated with the crossover described above in the section treated on the transport properties, while that of $45 \mathrm{~K}$-one, extended in a wide temperature range and having an amplitude about $1.3 \mathrm{~J} \mathrm{~K}^{-1} \mathrm{~mol}^{-1}$, which is close to that expected for $S=1 / 2$ case of a Kondo-lattice effect $C_{K}$ [56]. In analogy to the situation found for Ce-based compounds, Rietschel et al. have analyzed the uranium heavy-fermion superconductors: $\mathrm{UB}_{13}, \mathrm{UPt}_{13}$, and $\mathrm{U}_{2} \mathrm{Zn}_{17}$ in a similar way [57]. The value of the determined above amplitude is several time too small to ascribe it to a Schottky-type anomaly. For uranium compounds, due to usually large splittings of the CEF levels one cannot expect such a well defined anomaly peak (e.g. see Ref. [58]). Taking into account the fact reported by Desgranges and Schotte [56] that $\mathrm{T}_{\max }=0.45$ 
$T_{K}$, where $T_{K}$ is a Kondo temperature, one finds $T_{K}$ for UCoGe being equal to about $100 \mathrm{~K}$. This value is just equal to about $2 \mathrm{~T}_{\text {coh }}$. In addition, the temperature variation of the magnetic entropy, $S_{m}$, is shown in the inset of figure $12(b)$. At $100 \mathrm{~K} \mathrm{~S}_{\mathrm{m}}$ is equal to about $0.3 \mathrm{R} \ln 2$.

Moreover, in figure 13 the $C_{p}$ vs. $T$ curve is drawn separately for ThNiGe which may allow to perform an analysis of the lattice contribution to the total $C_{p}(T)$ dependence just in UCoGe, assuming that for this aim the Th-based compound as a non-magnetic reference may be used. As seen from this figure, only by combining the Debye and Einstein modes together with the electronic heat contribution we could fit fairly well the experimental data, by a smooth curve through the experimental points, mentioned above. Due to this fitting we got a little higher overall $\gamma_{\mathrm{ht}}$ than $\gamma(0)$. However, no satisfactory agreement between the fitting results and the experimental data has been obtained (not shown) using only the Debye approach. This is better apparent in figure 14 where we have plotted the $\mathrm{C}_{\mathrm{p}} / \mathrm{T}^{3}$ against $\mathrm{T}$ dependence for both ternaries considered here. It is well seen there that the low-temperature Einstein modes in the lattice heat do exist undoubtedly (see the appropriate maximum at $\mathrm{T}_{\max }$ for the Th-based material, while for the U-based one there is only a marked hump centered at the same $\mathrm{T}_{\max }$ being less apparent due to a large value of the electronic specific heat contribution found for the latter compound). This supports again the fact that the lattice dynamics of both these compounds are similar to each other. From rough fitting (solid line) we have obtained the approximate weighted equation, displayed in this figure, where $C_{D}\left(C_{E}\right)$ represents the specific heats due to the Debye (Einstein) phonons (involving the total nine expected modes, i.e., three acoustic and six optic modes) and with the electronic term $\mathrm{C}_{\mathrm{el}}$ also added. The corresponding 
values of the characteristic temperatures $\Theta_{\mathrm{D}}$ and $\Theta_{\mathrm{E}}$ are also given in this figure. It is thus clear from the above formula that for the best possible fitting, the lattice heat is found to require about seven oscillators per f.u. for the Debye phonons and about two oscillators per f.u. for the Einstein low-lying optic phonons. This procedure is taken from Ref. [59] where the case of YbGaGe was analyzed. Of course, as mentioned already above, the fitted electronic contribution is here represented by a high temperature coefficient, $\gamma_{\mathrm{ht}}$, which is a little bit higher than $\gamma(0)$ of ThNiGe.

\section{Conclusions}

Our results of bulk measurements done on an annealed single crystal show superconductivity but do not reproduce the previously reported coexistence with ferromagnetism. The magnetization studies, however, show the presence of lowtemperature strong ferromagnetic correlations in UCoGe, possibly due to the proximity of this compound into the quantum critical point. The recent paper by Slooten et. al. [18] has shown that near the ferromagnetic critical point $\mathrm{p}_{\mathrm{c}}=1.4 \mathrm{GPa}$ superconductivity is not only enhanced but also it is still present in the paramagnetic region induced by higher pressures. Thus, this result clearly indicates again the possibility for UCoGe to show up superconductivity without weak FM order.

The most essential to underline here is the fact that the electrical resistivity data exhibit a linear behavior at low temperatures for our non-magnetic sample of UCoGe being followed by the coherence-type knee in $\rho(\mathrm{T})$ at around $50 \mathrm{~K}$ and then at much higher temperatures this dependence reveals a single-ion Kondo-like effect. In turn, the transverse magnetoresistivity contains only a negative contribution in its field and temperature dependences in the whole range of the paramagnetic state of 
UCoGe and by this the presence of the Kondo-like state is confirmed in this germanide at higher temperatures. This state is also reflected by the corresponding high temperature Kondo- peak in the specific heat. The positive extremum in thermoelectric power, the maxima in the temperature derivatives of the resistivity and magnetoresistivity and also a low-temperature anomaly in the specific heat, all these occur almost at the same temperature of around $13 \mathrm{~K}$. Thus, this temperature gives rise to the crossover transition in UCoGe from a more localized behavior of the 5felectron states in the higher temperature region and, hence, the appearance of a Kondo-like effect to a more metallic state in the lower temperature region allowing for the superconductivity. Finally, the measured thermal conductivity points to the fact of a predominant role of the lattice contribution in $\mathrm{UCoGe}$, which may play some special role in both the exhibited superconductivity and thermopower behavior. Nevertheless, the remarkable large values of the upper critical field $\mathrm{B}_{\mathrm{C} 2}(0)$ ppovides evidence for spin-triplet superconductivity which persists in the paramagnetic phase of the considered here germanide [18].

\section{Acknowledgments}

We are gratefud to B. Coqblin for his interest in this paper and E. Talik for synthesizing the single crystal. Technical assistance of G. Badurski and R. Gorzelniak with some experiments as well as a fitting of the thermal conductivity by J. Mucha are acknowledged.

\section{References}

[1] R. Troć and V.H. Tran, J. Magn. Magn. Mater. 73 (1988) p. 389.

[2] F.R. de Boer, E. Brück, V. Sechovský, L. Havela and K.H.J. Buschow, Physica B 163 (1990) p. 175.

[3] L. Havela, A. Kolomiets, V. Sechovský, M. Diviš, M. Richter and A.V. Andreev, J. Magn. Magn. Mater 177-181 (1998) p. 47.

[4] K.H.J. Busahow, E. Brück, R.G. vaN Wierst, F.R. de Boer, L. Havela, V. Sechovský, P. Nozar, E. Sugiura( M. Ono, M. Date and Y. Yamagishi, J. Appl. Phys. 67 (1990) p. 5215.

[5] G.G, Lonzarich, in Electron: A Centenary Volume, M. Springford, ed., Cambridge Univ. Press\$ Cambridge ̌̌ 199, Chap. 6\&

[6] E. Diviš, Physica B 403 (2008) p. 2505.

[7] P. de la Mora and O. Lavarro\$ J. Phys.: Condens. Matter 20 (2008) p. 285221.

[8] M. Samsel-Czekała, S. Elgazzar, P.M. Oppeneer, E. Talik, W. Walerczyk and R. Troć, J. Phys. 
Condens. Matter. 22 (2010) p. 015503.

[9] FPLO-5.00-18 and 5.10-20 [improved version of the original FPLO code by K. Koepernik and H. Eschrig, Phys. Rev. B 59 (1999) p. 1743]; http://www.FPLO.de.

[10] N.T. Huy, A. Gasparini, D.E. de Nijs, Y. Huang, J.C.P. Klaasse, T. Gortenmulder, A. de Visser, A. Hamann, T. Görlach and H. v. Löhneysen, Phys. Rev. Lett. 99 (2007) p. 067006.

[11] S. Sakarya, N.T. Huy, N.H. van Dijk, A. de Visser, M. Wagemaker, A.C. Moleman, T.J. Gortenmulder, J.C.P. Klaasse, M. Uhlarz and H. v. Löhneysen, J. Alloys Compd. 457 (2008) p. 51.

[12] D.E. de Nijs, N.T. Huy and A. de Visser, Phys. Rev. B 77 (2008) p. 140506(R).

[13] N.T. Huy, D.E. de Nijs, Y.K. Huang and A. de Visser, Phys. Rev. Lett. 100 (2008) p. 077002.

[14] E. Hassinger, D. Aoki, G. Knebel and J. Flouquet, J. Phys. Soc. Jpn. 77 (2008) p. 073703.

[15] A. de Visser, N.T. Huy, A. Gasparini, D.E. de Nijs, D. Andreica, C. Baines and A. Amato, Phys. Rev. Lett. 102 (2009) p. 167003.

[16] T. Ohta, Y. Nakai, Y. Ihara, K. Ishida, K. Deguchi, N. K. Sato and I. Satoh, J. Phys. Soc. Jap. 77 (2008) p. 023707.

[17] N.T. Huy, Y.K. Huang and A. de Visser, J. Magn. Magn. Mater. 321 (2009) p. 2691.

[18] E. Slooten, T. Naka, A. Gasparini, Y.K. Huang and A. de Visser, Phys. Rev. Lett. 103 (2009) p. 097003.

[19] R. Wawryk and Z. Henkie, Phil. Mag. B 81 (2001) p. 223.

[20] Z. Henkie and R. Wawryk, Solid State Commun. 122 (2002) p. 1.

[21] J.P. Perdew and Y. Wang, Phys. Rev. B 45 (1992) p. 13244.

[22] V. Sechovsky, L. Havela, F.R. de Boer, J.J.M. Franse, P.A. Veenhuizein, J. Sebec, J. Stechno, A.V. Andreev, Physica 142B (1986) p. 283.

[23] V.H. Tran, R. Troć, H. Noël, J. Alloys Compd. 262-264 (1997) p. 471.

[24] D. Kaczorowski, H. Noël, J. Phys.: Condens. Matter 5 (1993) p. 9185.

[25] N.T. Huy and A. de Visser, Solid State Commun. 149 (2009) p. 703.

[26] T.D. Matsuda, D. Aoki, S. Ikeda, E. Yamamoto, Y. Haga, H. Ohkuni, R. Settai and Y. Ōnuki, Proc. J. Phys. Soc. Jpn. 77, Suppl A (2008) p. 362.

[27] D. Aoki, Y. Haga, T.D. Matsuda, S. Ikeda, Y. Homma, H. Sakai, Y. Shiokawa, E. Yamamoto, N. Nakamura, R. Settai and Y. Ōnuki, J. Phys.: Condens. Matter 21 (2009) p. 164203.

[28] W. Miiller and V.H. Tran, Acta Phys. Pol. A 115 (2009) p. 144.

[29] D.R. Grempel and M.J. Rozenberg, Phys. Rev. B 60 (1999) p. 4702.

[30] N.T. Huy, A. Gasparini, J.C.P. Klaasse, A. de Visser, S. Sakarya and N.H. Van Dijk, Phys. Rev. B. 75 (2007) p. 212405.

[31] P. Manfrinetti, A. Palenzona, S.K. Dhar and C. Mitra, Intermetallics 7 (1999) p. 1291.

[32] As an example, see J.S. Schilling, Phys. Rev. B 33 (1986) p. 1667.

[33] N.F. Mott, Proc. Roy. Soc. 156 (1936) p. 368.

[34] T.T.M. Palstra, A.A. Menovsky and J.A. Mydosh, Phys. Rev. B 33 (1986) p. 6527.

[35] F. Canepa, P. Manfrinetti, M. Pani and A. Palenzona, J. Alloys Compd. 234 (1996) p. 225.

[36] V. Zlatić, T.A. Costi, A.C. Hewson and B.R. Coles, Phys. Rev. B 48 (1993) p. 16152; D.L. Cox and N. Grewe, Z. Phys. B 71 (1988) p. 321.

[37] F.J. Blatt, in Physics of Electronic Conduction in Solids, McGraw-Hill, New York, 1968.

[38] A. de Visser, J.J.M. France and A. Menovsky, J. Magn. Magn. Mater. 43 (1984) p. 43.

[39] F. Steglich, U. Rauchschwalbe, U. Gottwick, H.M. Mayer, G. Sparm, N. Grewe, U. Poppe and J.J.M. Franse, J. Appl. Phys. 57 (1985) p. 3054.

[40] T. Takabatake, S. Miyata, H. Furii, Y. Aoki, T. Suzuki, T. Fujita, J. Samuraj and T. Hiraoka, J. Phys. Soc. Jpn. 59 (1990) p. 4412.

[41] U. Gottwick, K. Gloos, S. Horn, F. Steglich and N. Grewe, J. Magn. Magn. Mater. 47-48 (1985) p. 536.

[42] F.J. Blatt, P.A. Schroeder, C.L. Foiles and D. Greig, Thermoelectric Power of Metals, Plenum, New York, 1976, p. 147, and references therein.

[43] R. Troć, Z. Bukowski, C. Sułkowski, H. Misiorek, J.A. Morkowski, A. Szajek and G. Chełkowska, Phys. Rev. B 70 (2004) p. 184443.

[44] H.J. Trodahl, Phys. Rev. B 51 (1995) p. 6175.

[45] Y. Bando, T. Suemitsu, K. Takagi, H. Tokushima, Y. Echizen, K. Katoh, K. Umeo, Y. Maeda and T. Takabatake, J. Alloys Compd. 313 (2000) p. 1. 
[46] E. Bauer, E. Gratz, G. Hutflesh, A.K. Bhattacharje and B. Coqblin, J. Mag. Mag. Mater. 108 (1992) p. 159.

[47] E. Gratz, E. Bauer, B. Barbara, S. Zemirli, F. Steglich, C.D. Bredl and W. Lieke, J. Phys. F: Metal Phys. 15 (1985) p. 1975.

[48] M. Hiroi, M. Sera, N. Kobayashi, Y. Haga, E. Yamamoto and Y. Onuki, J. Phys. Soc. Jpn. 66 (1997) p. 1595.

[49] W. Franz, A. Grießel, F. Steglich and D.Wohlleben, Z. Phys. B 31 (1978) p. 7.

[50] I.A. Smirnov and V.S. Oskotski, in Handbook on the Physics and Chemistry of Rare Earths, Vol. 16, K.A. Gschneidner and L. Eyring, eds., North Holland, Amsterdam 1993, p. 116.

[51] R. Berman, in Thermal Conductivity in Solids, Carendon, Oxford, 1976.

[52] J. Callaway, Phys. Rev. 113 (1959) p. 1046.

[53] J. Mucha, H. Misiorek, R. Troć and B. Coqblin, J. Phys.: Condens. Matter 20 (2008) p. 085205.

[54] J. Mucha, H. Misiorek, R. Troć and Z. Bukowski, J. Phys.: Condens. Matter 18 (2006) p. 3097.

[55] S. Kawamata, H. Iwaszki and N. Kobayashi, J. Magn. Magn. Mater. 104-107 (1992) p. 55.

[56] H.U. Desgranges and K.D. Schotte, Phys. Lett. 91A (1982) p. 240.

[57] H. Rietschel, B. Renker, R. Felten, F. Steglich and G. Weber, J. Magn. Magn. Mater. $76-77$ (1988) p. 105.

[58] G. Amoretti, A. Blaise, J.M. Collard, R.O.A. Hall, M.J. Mortimer and R. Troć, J. Magn. Magn. Matter. 46 (1984) p. 57.

[59] K-H. Jang, W-Y Song, J.-G. Park, H.C. Kim, A. Lindbaum, H. Müller and E. Bauer, Europhys. Lett. 69 (2005) p. 88.

\section{Figure captions}

Figure 1. (Color online) Part (a): Magnetic susceptibility $\chi_{i}$ against temperature for as grown and annealed samples of UCoGe. The inset shows the isothermal magnetization $\mathrm{M}$ vs. an applied magnetic field. Part (b): Arrott plot of magnetisation isotherms corresponding to M curves displayed in the inset to part (a).

Figure 2. (Color online) The inverse magnetic susceptibility $\chi^{-1}$ of the UCoGe crystal measured along two directions (indicated) as a function of temperature. The insets show the magnetization $\mathrm{M}$ against both temperature $\mathrm{T}$ (upper one) and applied magnetic fields $\mathrm{B}$ at $1.72 \mathrm{~K}$ (lower one) for both crystallographic directions The latter was measured in increasing (open symbols) and decreasing (solid symbols) fields.

Figure 3. (Color online) Electrical resistivity $\rho(\mathrm{T})$ curves for as grown and annealed samples of UCoGe. Left hand inset shows the low temperature behavior of $\rho(T)$, and right hand one presents the temperature derivative $\mathrm{d} \rho(\mathrm{T}) / \mathrm{dT}$ of the resistivity.

Figure 4. (Color online) The low temperature resistivity on the expanded temperature scale.

Figure 5. (Color online) The determination of the differential resistivity $\Delta \rho_{1,2}\left(=\Delta \rho_{1}\right.$ $\Delta \rho_{2}$ ) against temperature for the annealed sample of UCoGe after subtracting the phonon reference data based on ThNiGe.

Figure 6. (Color online) The differential resistivity $\Delta \rho_{1,2}\left(=\Delta \rho_{1}-\Delta \rho_{2}\right)$ as in figure 5 vs. temperature on a logarithmic scale. The inset displays a fitting of the experimental resistivity taken for ThNiGe to the Bloch-Grüneisen-Mott equation [33]. 
Figure 7. (Color online) The low-temperature dependences of the resistivity in $\mathrm{B}=0$ and $8 \mathrm{~T}$ (a) and their transverse magnetoresistivity $\Delta \rho / \rho_{0}(\mathrm{~T})$ at $\mathrm{B}=8 \mathrm{~T}$ (b) for the annealed sample of UCoGe. The insets illustrate the Fermi-liquid behavior of the resistivity measured at $8 \mathrm{~T}$ (a) and the temperature derivative of the magnetoresistivity, $d\left(\Delta \rho / \rho_{0}(T)\right) / d T$, at $B=8 \mathrm{~T}(b) . T_{\text {inf }}(=13 \mathrm{~K})$ is the characteristic temperature of the crossover (see text). The solid squares indicate the values of TMR taken from figure 8 at $8 \mathrm{~T}$.

Figure 8. (Color online) Transverse magnetoresistivity against applied magnetic fields $\Delta \rho / \rho_{0}$ (B) up to $8 \mathrm{~T}$ measured at various temperatures $4.2-40 \mathrm{~K}$. The dashed curves depict the square $B^{2}$ field dependencies. In the inset the coefficient $\alpha$ is plotted against $\mathrm{T}$.

Figure 9. (Color online) Measured (symbols) and calculated (dashed line) TEP for UCoGe. The upper inset shows the low temperature $S(T)$ dependences for two samples of UCoGe: as grown and annealed (open and solid circles, respectively) and, additionally, the first temperature derivative of $\mathrm{S}(\mathrm{T})$ for the latter sample (squares). One can see from this inset an almost linear variation of $S(T)=\alpha \mathrm{T}$ below about $2.5 \mathrm{~K}$. The lower inset displays a fitting of the phenomenological modified Mott equation to the low temperature variation of $\mathrm{S}(\mathrm{T})$.

Figure 10. (Color online) The calculated two parts of densities of states DOS in paramagnetic UCoGe, i.e., $\mathrm{N}_{s}(\mathrm{E})$ and $\mathrm{N}_{p d f}(\mathrm{E})$, defined in the text. The insert shows the energy derivative of $\mathrm{N}_{p d f}(\mathrm{E})$ drawn in the enlarged scale around $\mathrm{E}_{\mathrm{F}}$.

Figure 11. (Color online) Thermal conductivity $\kappa_{\text {tot }}$ vs. T (squares). After using the W-F law and making separation of $\kappa_{\text {tot }}$, we obtained its two main contributions: electronic $\kappa_{\mathrm{e}}$ (dotted curve) and lattice $\kappa_{\text {lat }}$ (exp.) (circles) parts, respectively. The dashed line presents the difference $\Delta \kappa_{\text {lat }}$ obtained by extracting from $\kappa_{\text {lat }}($ exp.) the $\kappa_{\mathrm{ph}}$ (calc.) part (solid line) based on the Calleway model which is discussed in the text. The inset shows the normalized $\mathrm{L} / \mathrm{L}_{0}$ ratio vs. $\mathrm{T}$.

Figure 12. (Color online) Part (a): Specific heat $C_{p}$ vs. $T$ for UCoGe and the reference compound $\mathrm{ThNiGe}$ as well as the simulated $\mathrm{C}_{\mathrm{ph}}(\mathrm{T})$ function, based on the latter compound. The lower and upper insets show, the low-temperature $C_{p} / T$ vs. $T^{2}$ curves for both compounds and $\mathrm{C}_{\mathrm{p}} / \mathrm{T}$ vs. $\mathrm{T}$ dependencies for only UCoGe, respectively. Part (b) The difference $\Delta \mathrm{C}_{\mathrm{p}}=\mathrm{C}_{\mathrm{p}}(\mathrm{UCoGe})-\mathrm{C}_{\mathrm{ph}}$ depending on $\mathrm{T}$, as specified in the text. In the inset the same dependence is displayed but with the high temperature electronic specific heat $\left(\mathrm{C}_{\mathrm{el}}=\gamma^{*} \mathrm{~T}\right)$ being subtracted. In addition, the temperature variation of the magnetic entropy $S_{m}$ of $U 5 f$ electrons is also presented (solid line).

Figure 13. (Color online) Total specific heat $\mathrm{C}_{\mathrm{p}}$ measured for $\mathrm{ThNiGe}$ (solid circles). A deconvolution of the total $C_{p}$ into the Debye $\left(C_{D}\right)$, Einstein $\left(C_{E}\right)$, and electronic $\left(\mathrm{C}_{\mathrm{el}}\right)$ specific heat contributions is also presented as $\mathrm{C}_{\mathrm{fit}}$ (solid line). The two former contributions define the smooth $\mathrm{C}_{\mathrm{ph}}(\mathrm{T})$ curve, shown in figure 12(a). The fitting parameters shown in the figure are described in the text. 
Figure 14. (Color online) The $\mathrm{C}_{\mathrm{p}} / \mathrm{T}^{3}$ vs. $\mathrm{T}$ plot presents all the specific heat contributions into $\mathrm{C}_{\mathrm{p}}$ of $\mathrm{ThNiGe}$, described in figure 13 and additionally such a total plot for UCoGe. 

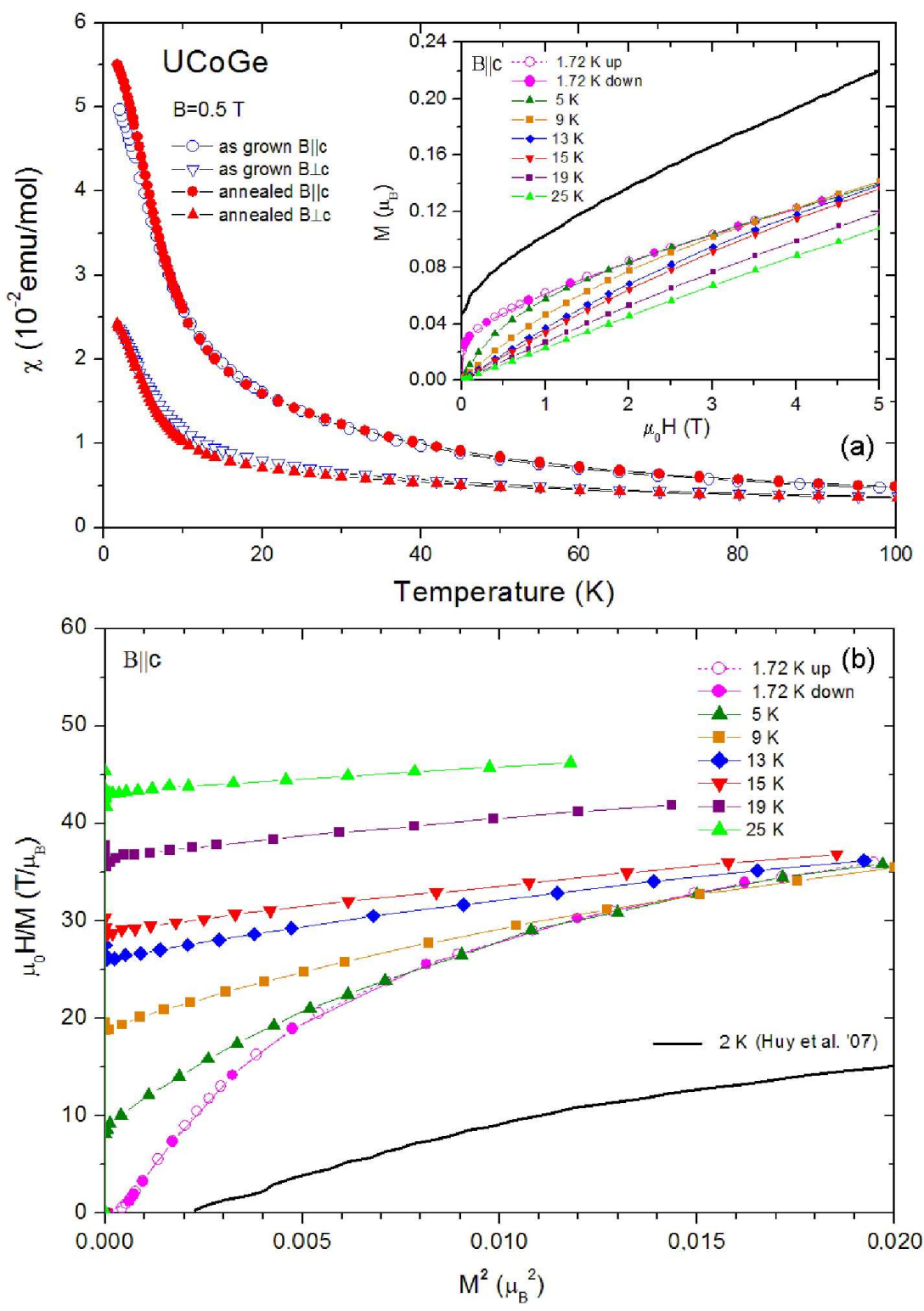

$378 \times 519 \mathrm{~mm}(600 \times 600 \mathrm{DPI})$

http://mc.manuscriptcentral.com/pm-pml 


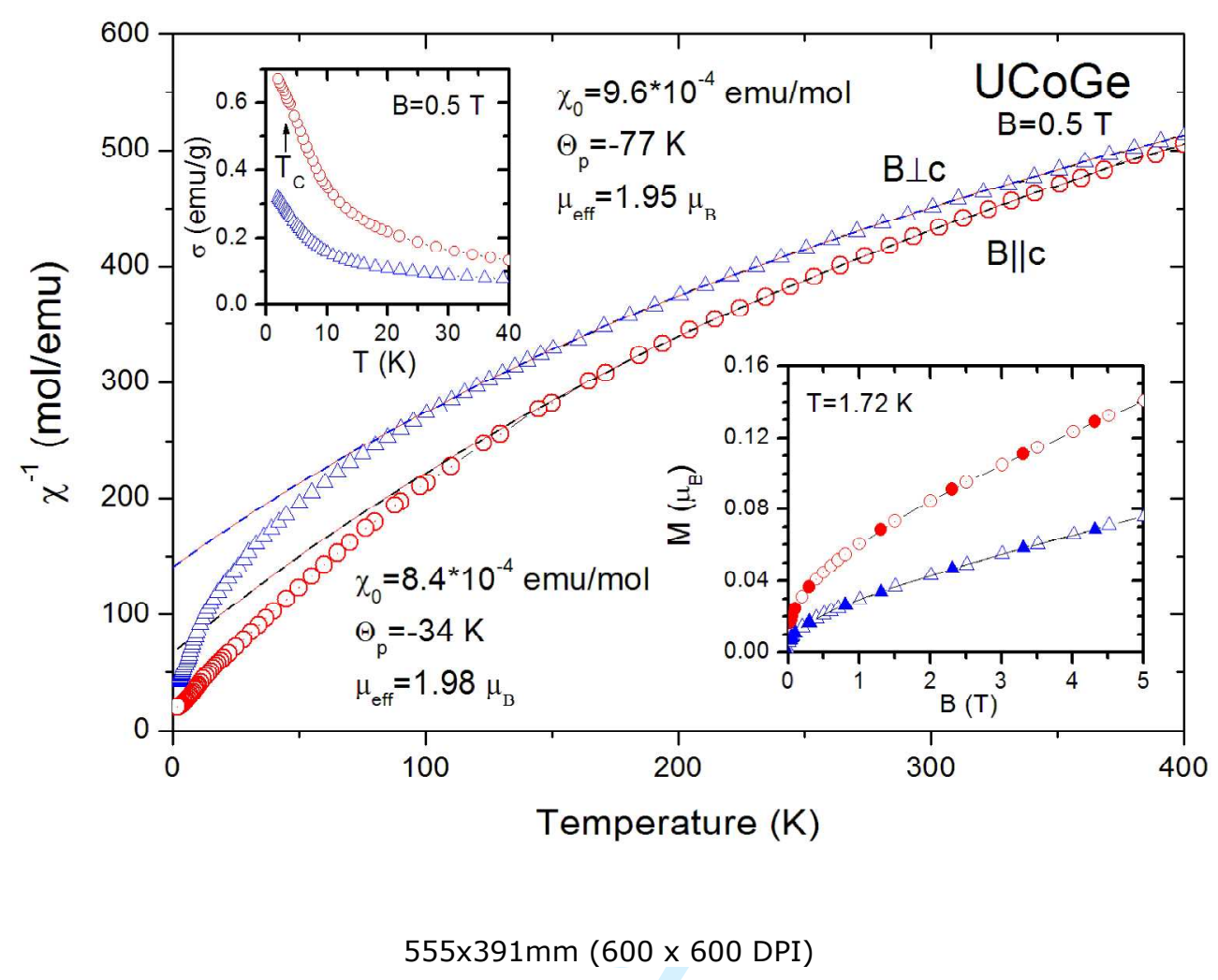

http://mc.manuscriptcentral.com/pm-pml 


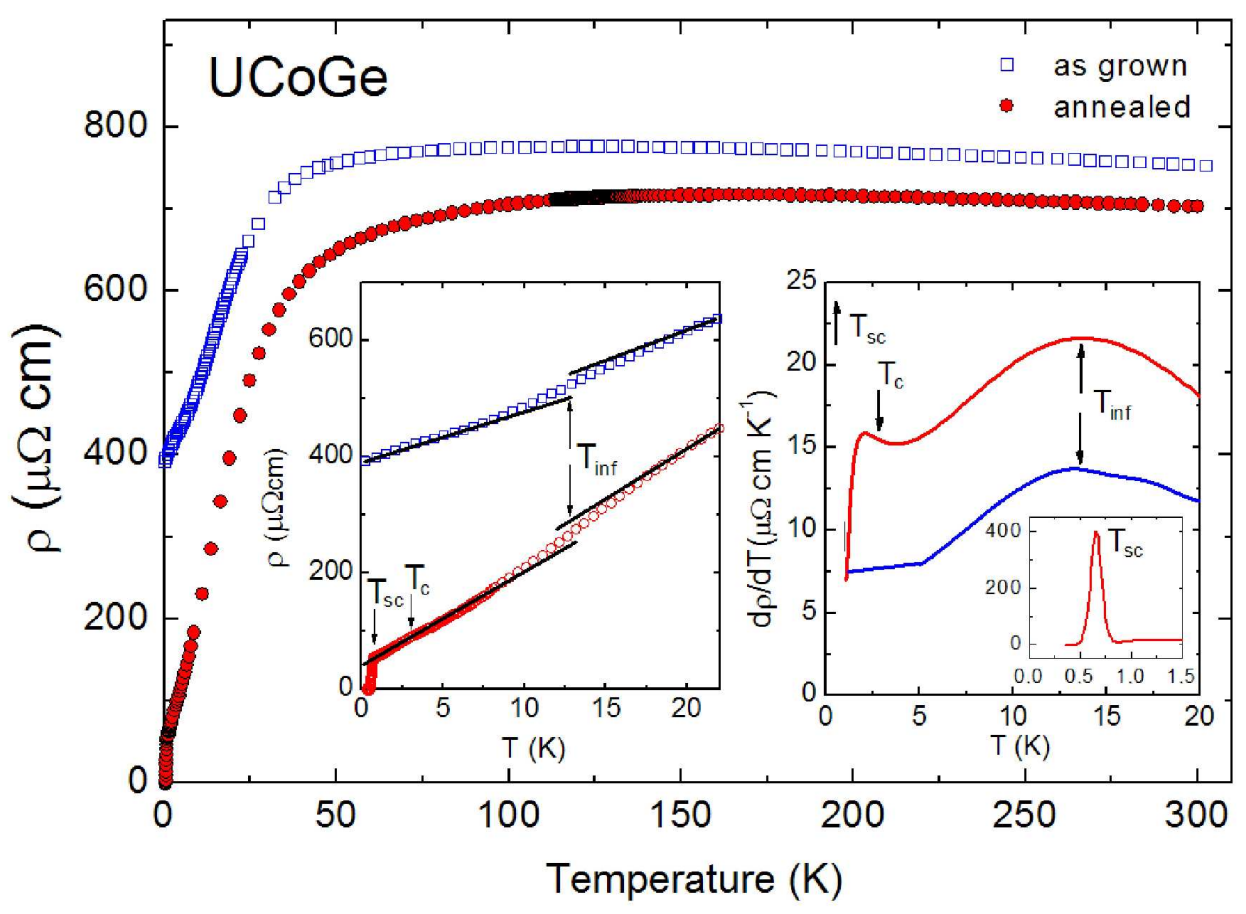

$388 \times 286 \mathrm{~mm}(600 \times 600 \mathrm{DPI})$ 


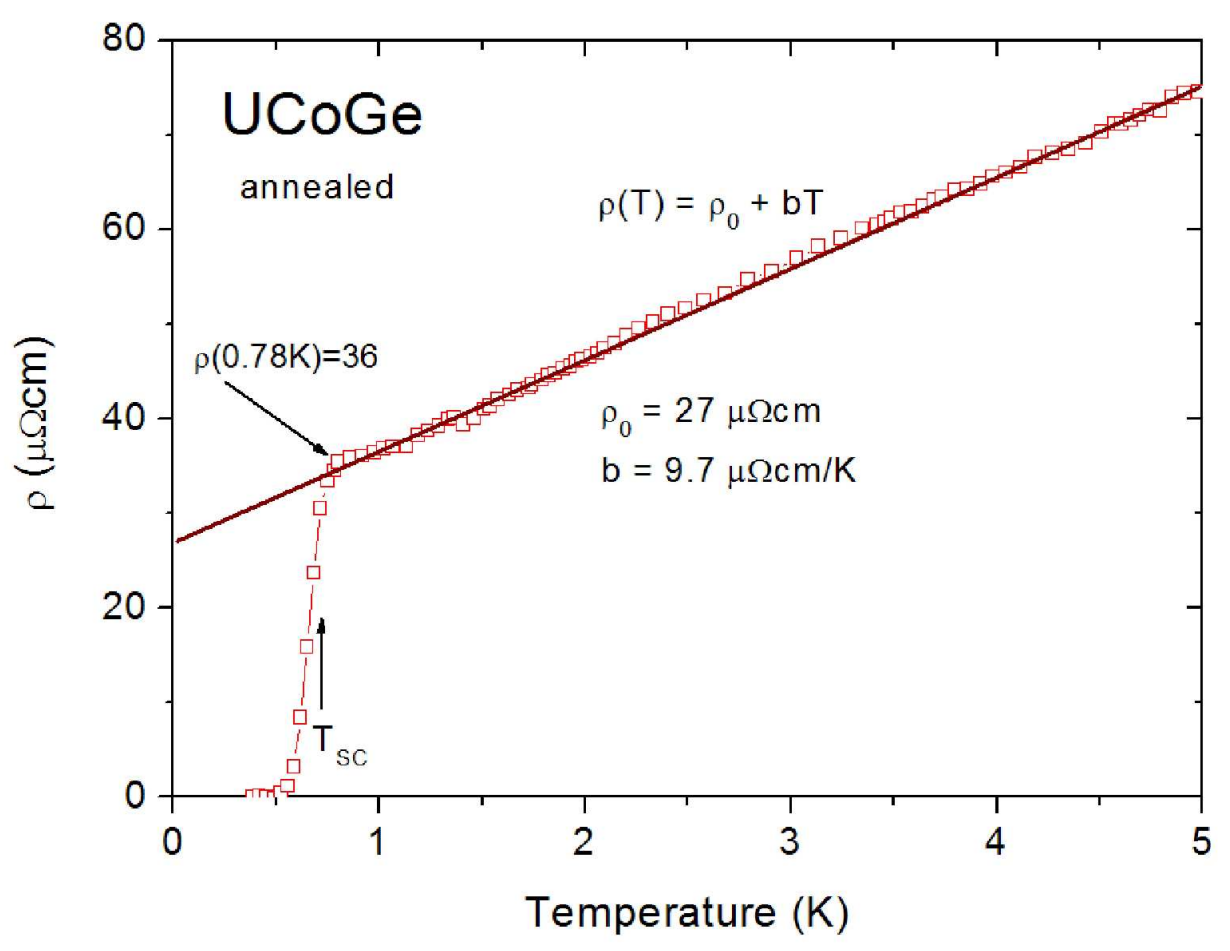

$345 \times 268 \mathrm{~mm}(600 \times 600 \mathrm{DPI})$ 


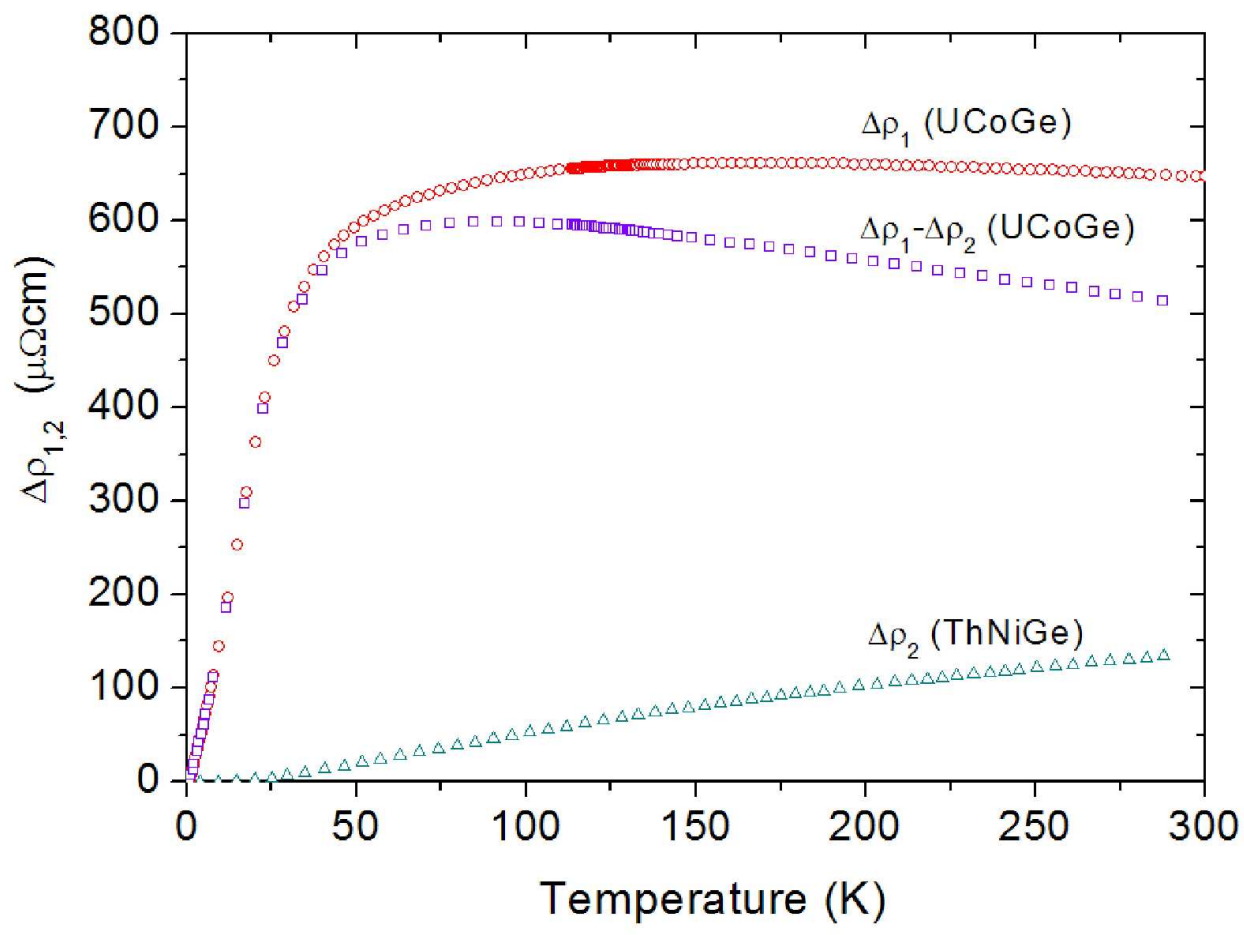

$349 \times 264 \mathrm{~mm}(600 \times 600 \mathrm{DPI})$ 


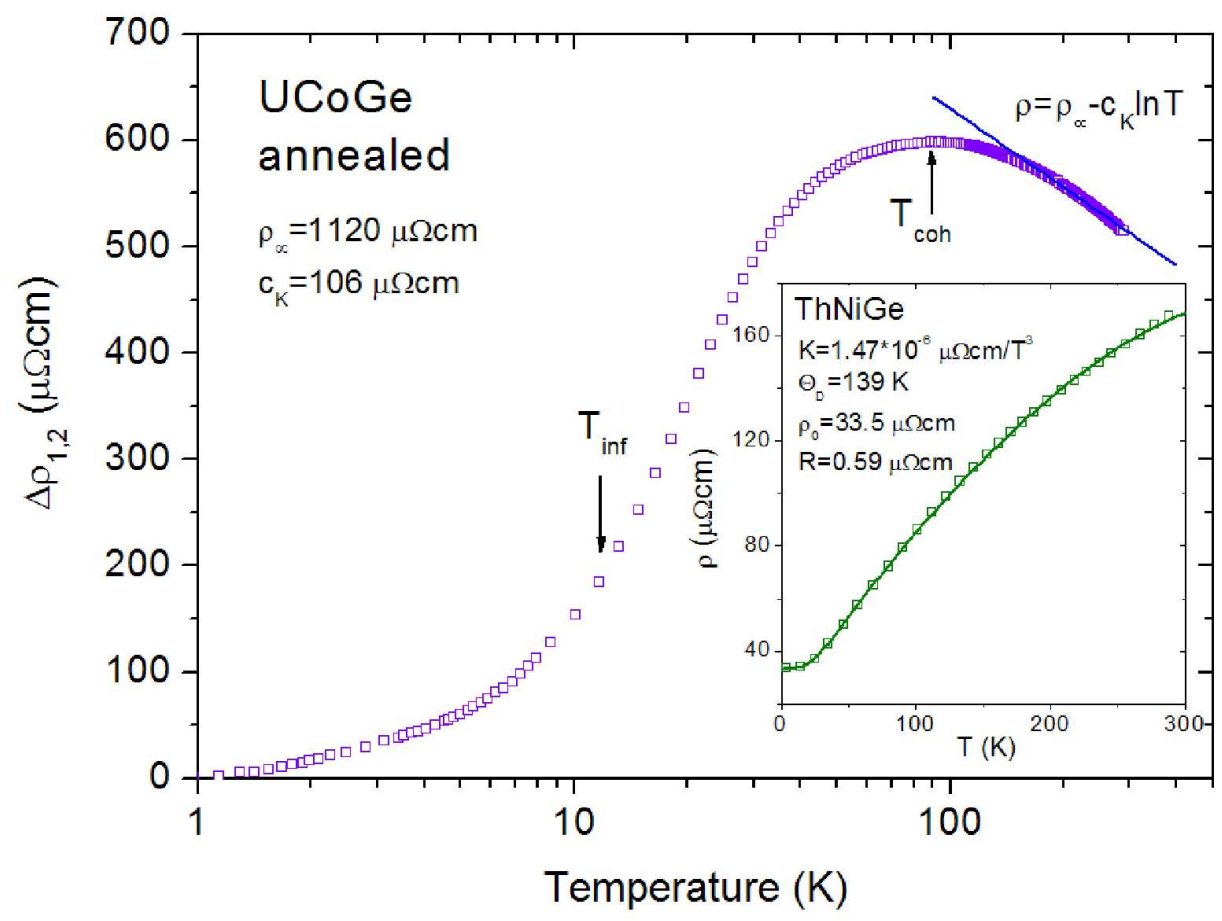

$350 \times 261 \mathrm{~mm}(600 \times 600 \mathrm{DPI})$ 

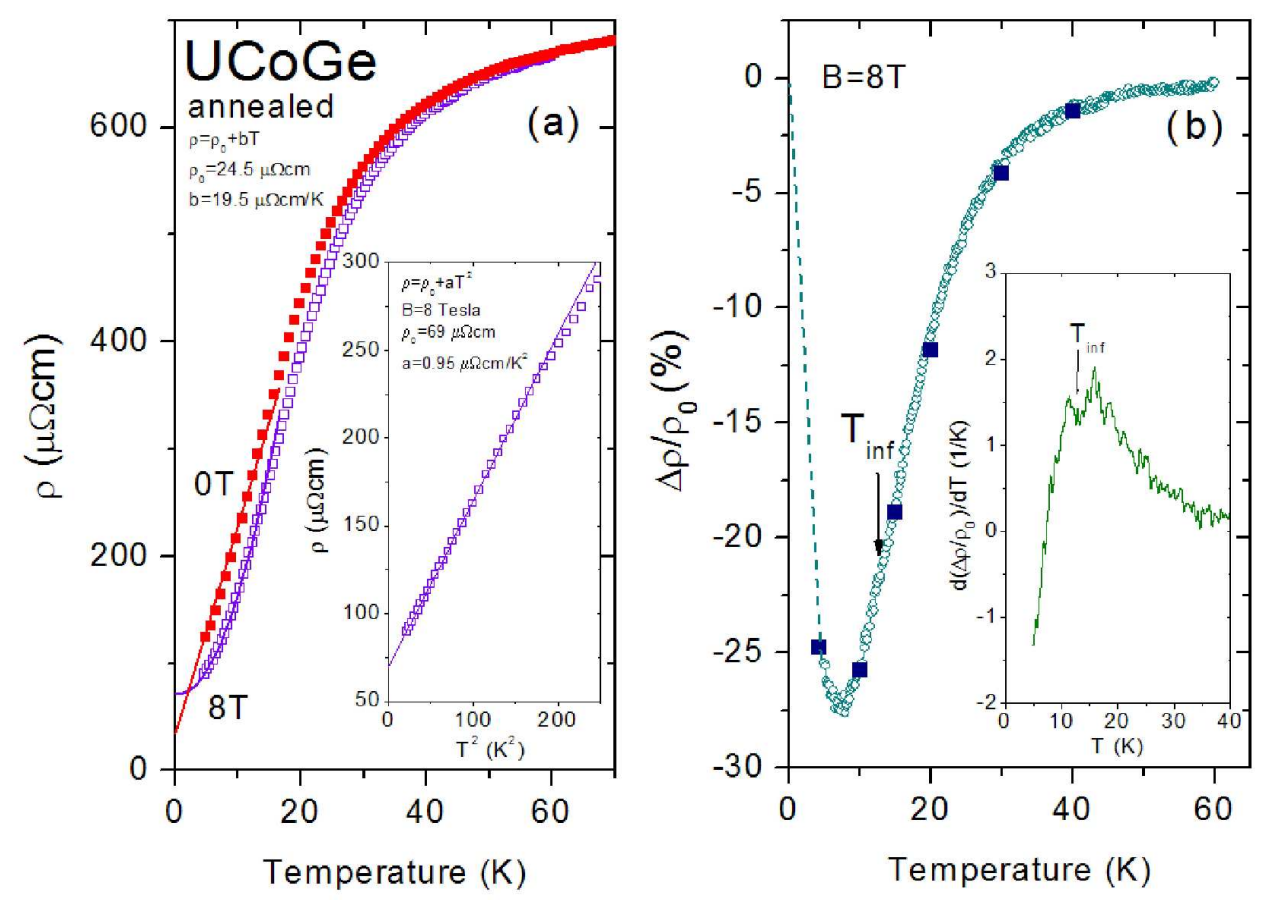

$362 \times 264 \mathrm{~mm}(600 \times 600 \mathrm{DPI})$

http://mc.manuscriptcentral.com/pm-pml 


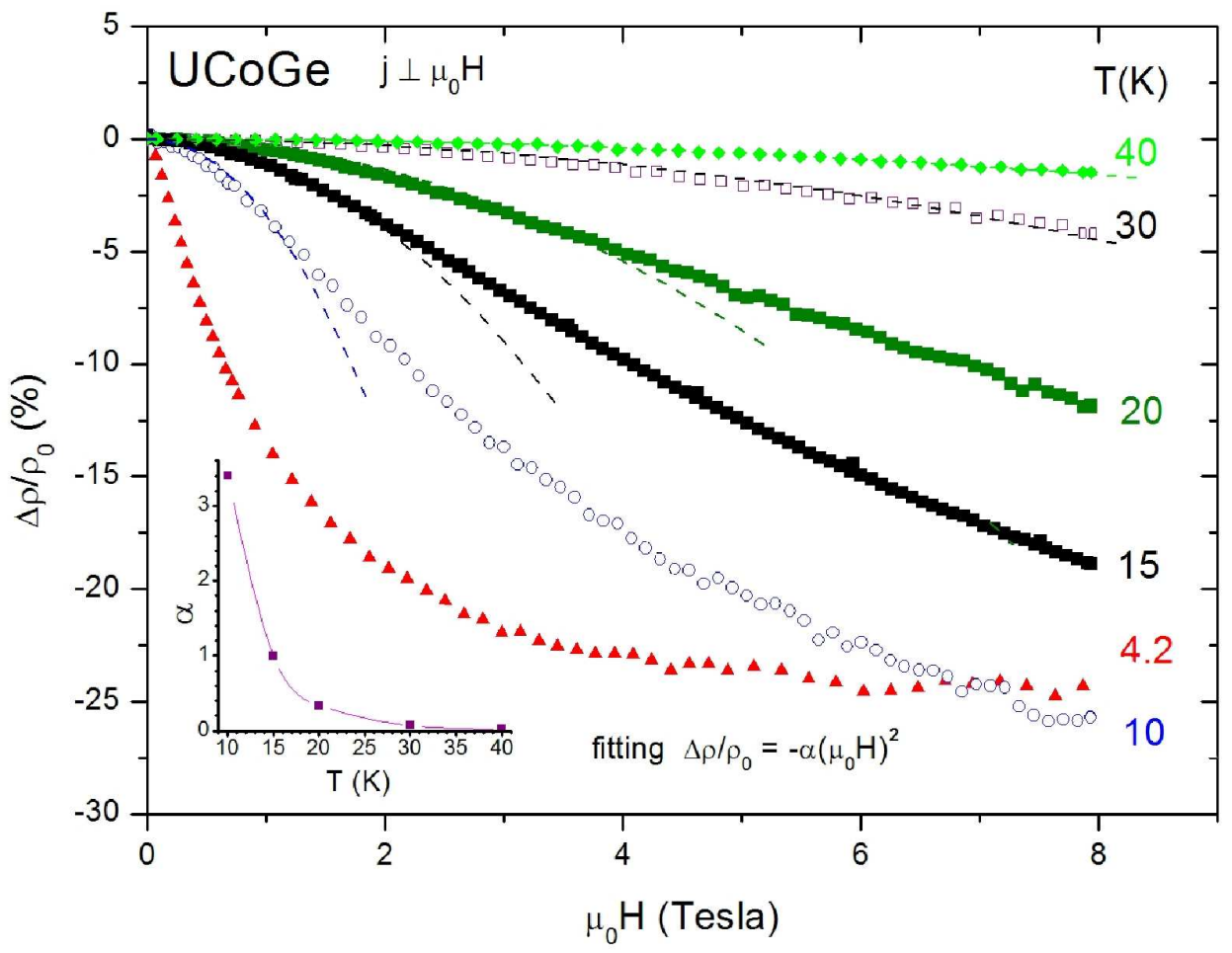

$492 \times 384 \mathrm{~mm}(600 \times 600 \mathrm{DPI})$ 


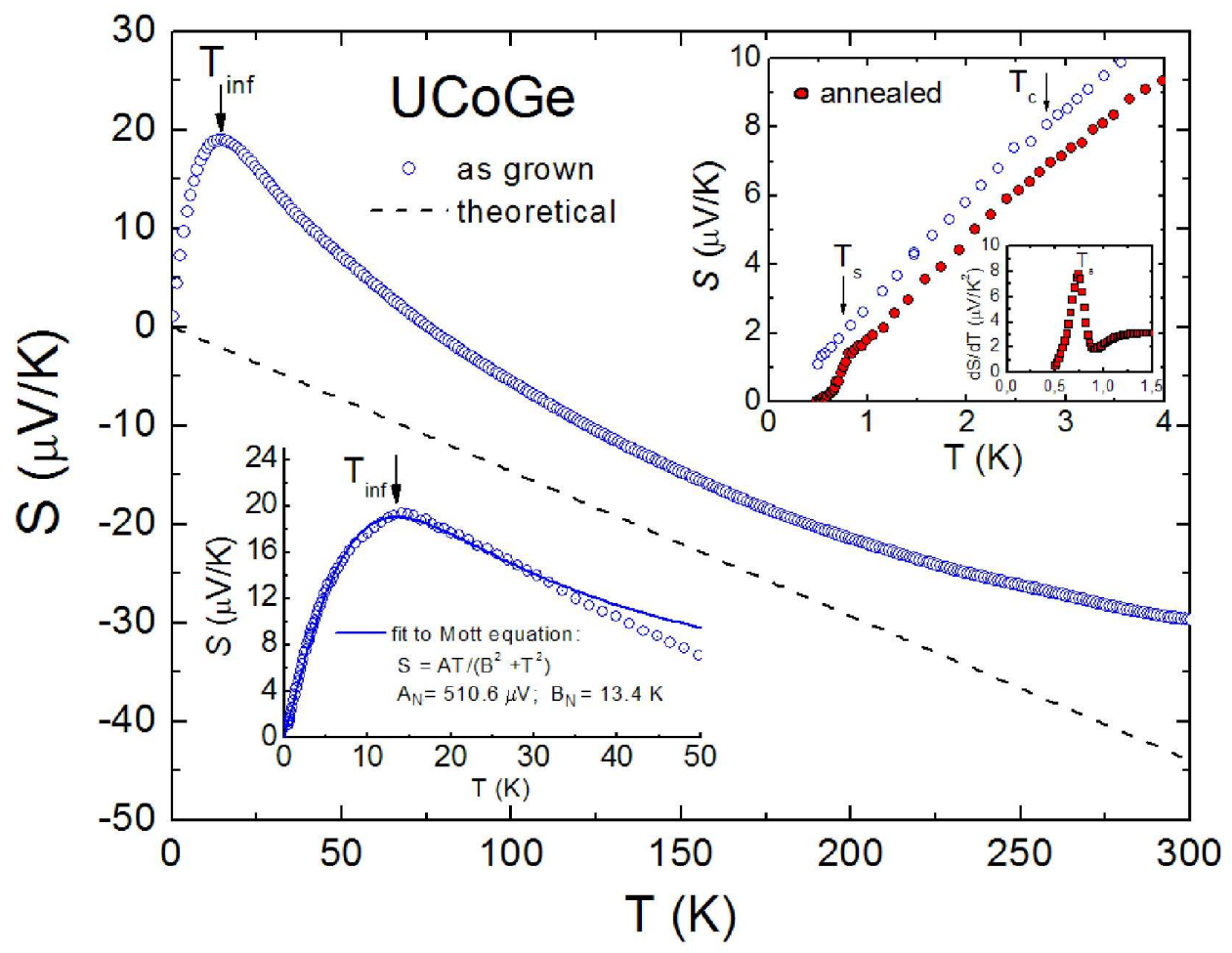

$369 \times 287 \mathrm{~mm}(600 \times 600 \mathrm{DPI})$ 


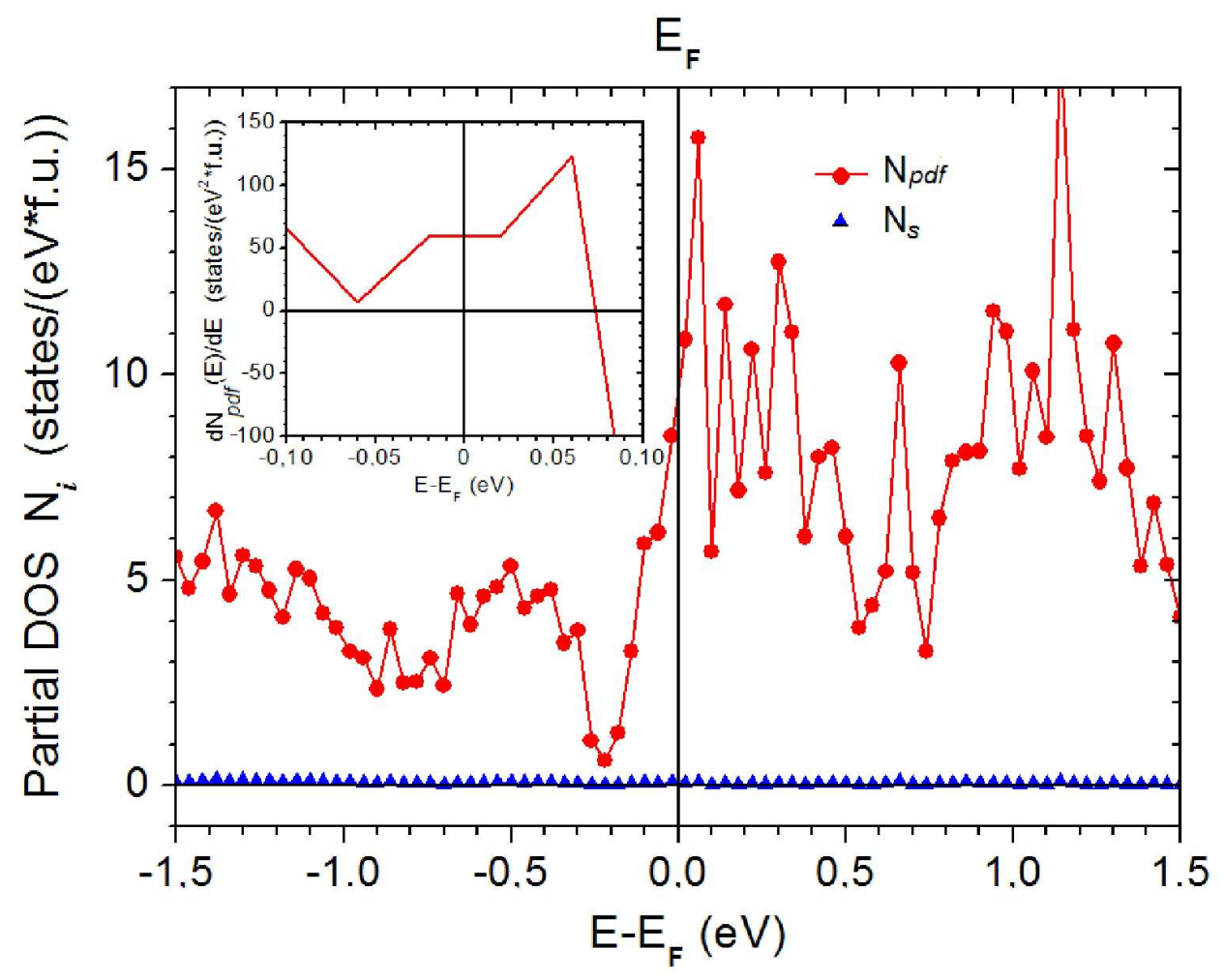

$337 \times 269 \mathrm{~mm}(600 \times 600 \mathrm{DPI})$ 


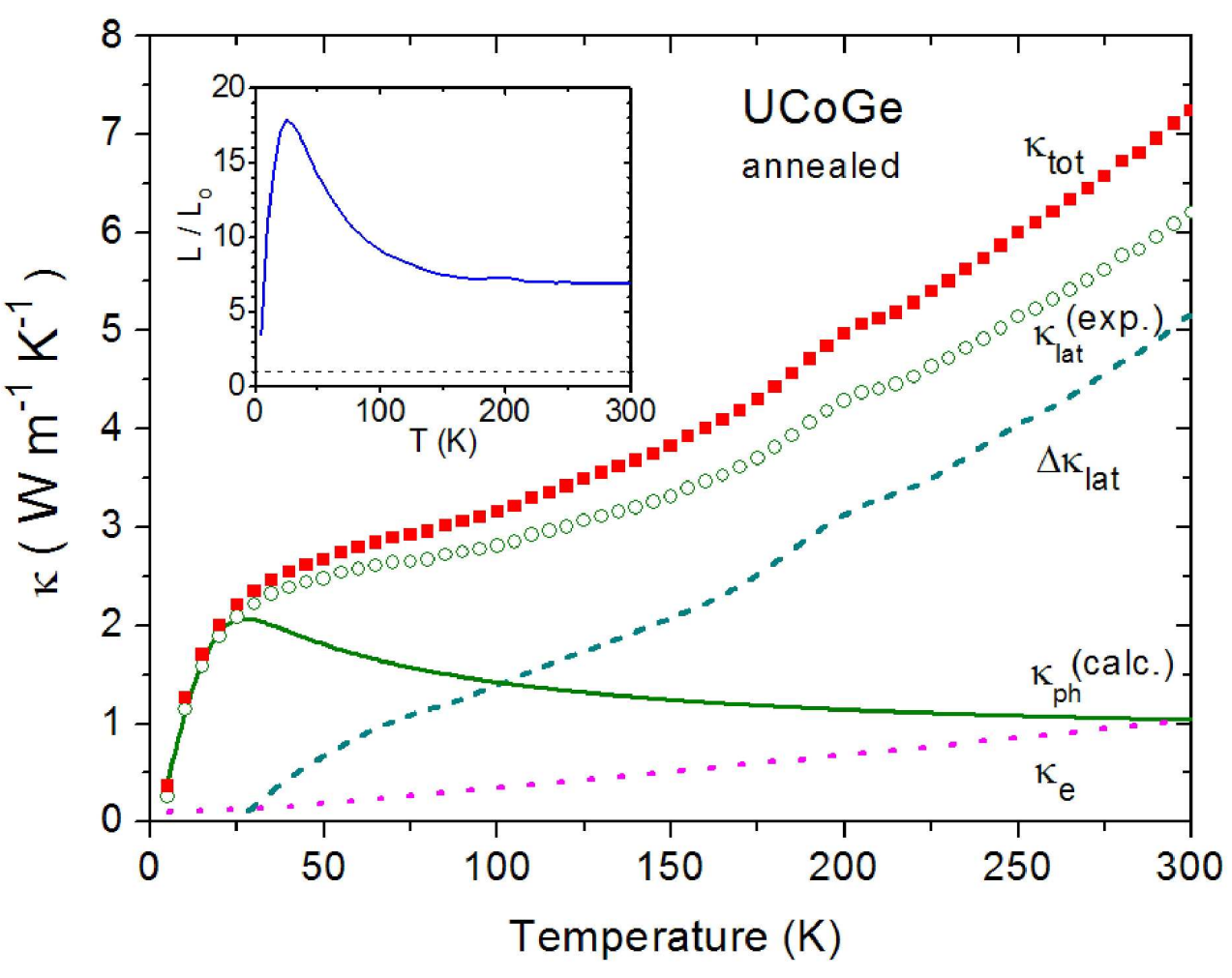

$324 \times 261 \mathrm{~mm}(600 \times 600$ DPI $)$ 


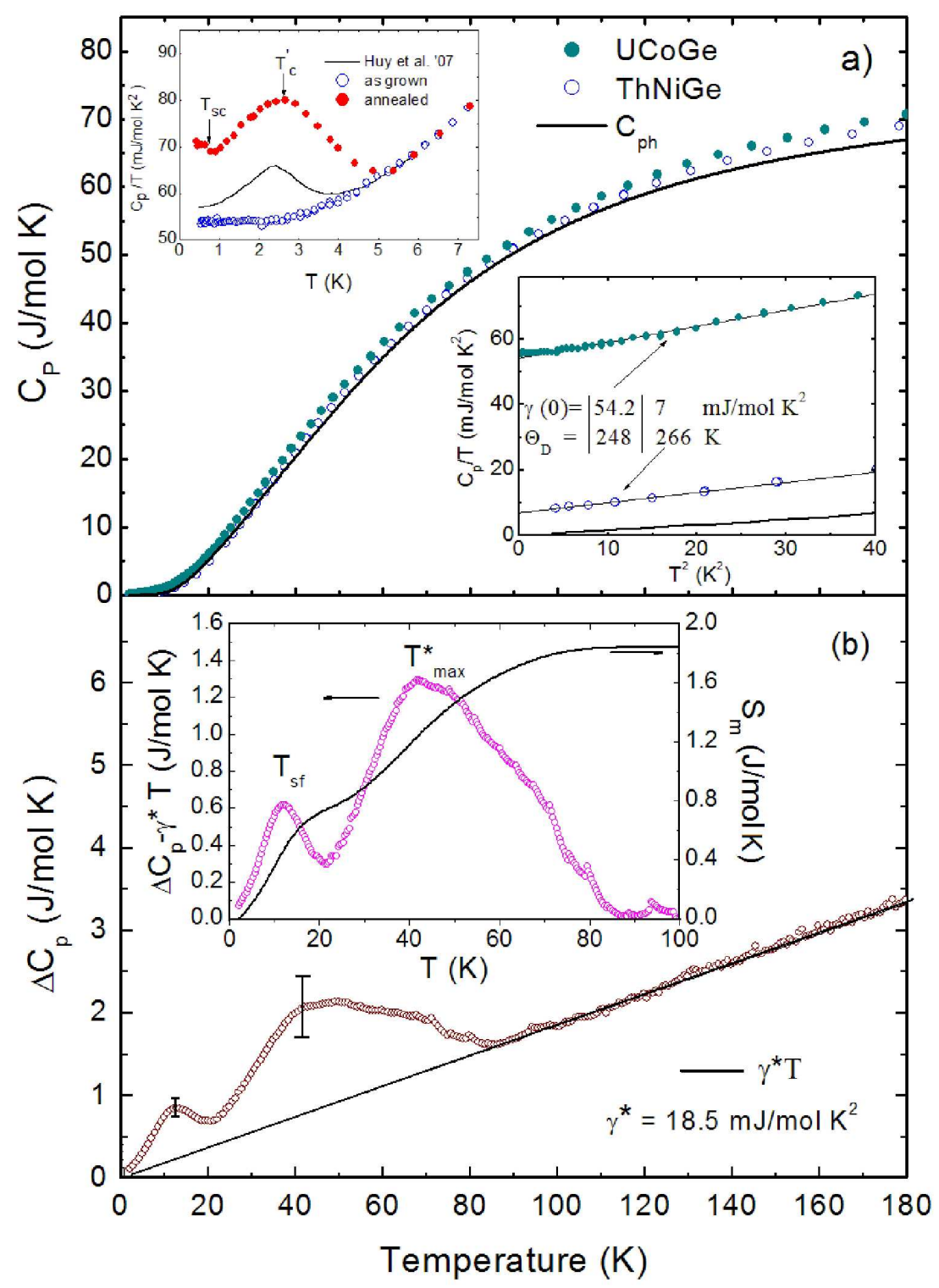

$334 \times 459 \mathrm{~mm}(600 \times 600 \mathrm{DPI})$

http://mc.manuscriptcentral.com/pm-pml 


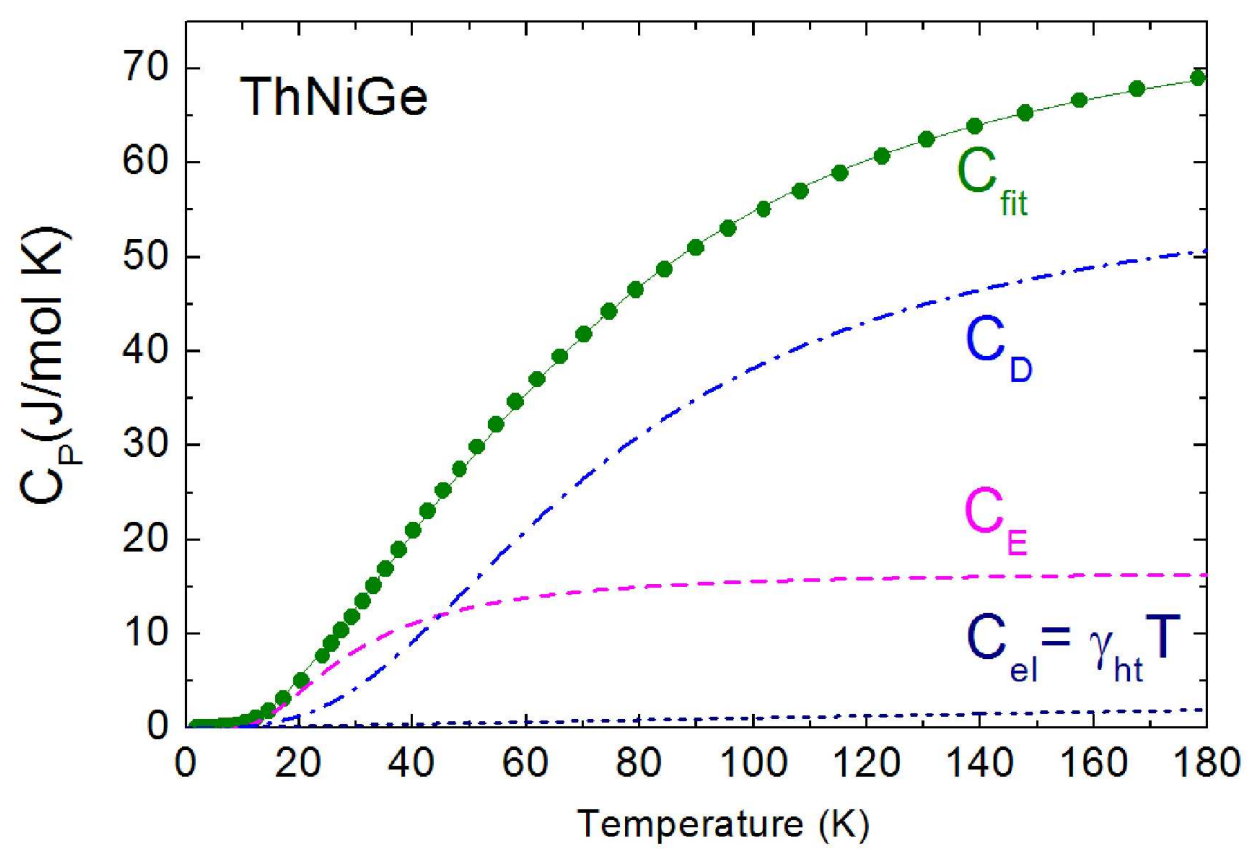

$382 \times 267 \mathrm{~mm}(600 \times 600 \mathrm{DPI})$ 


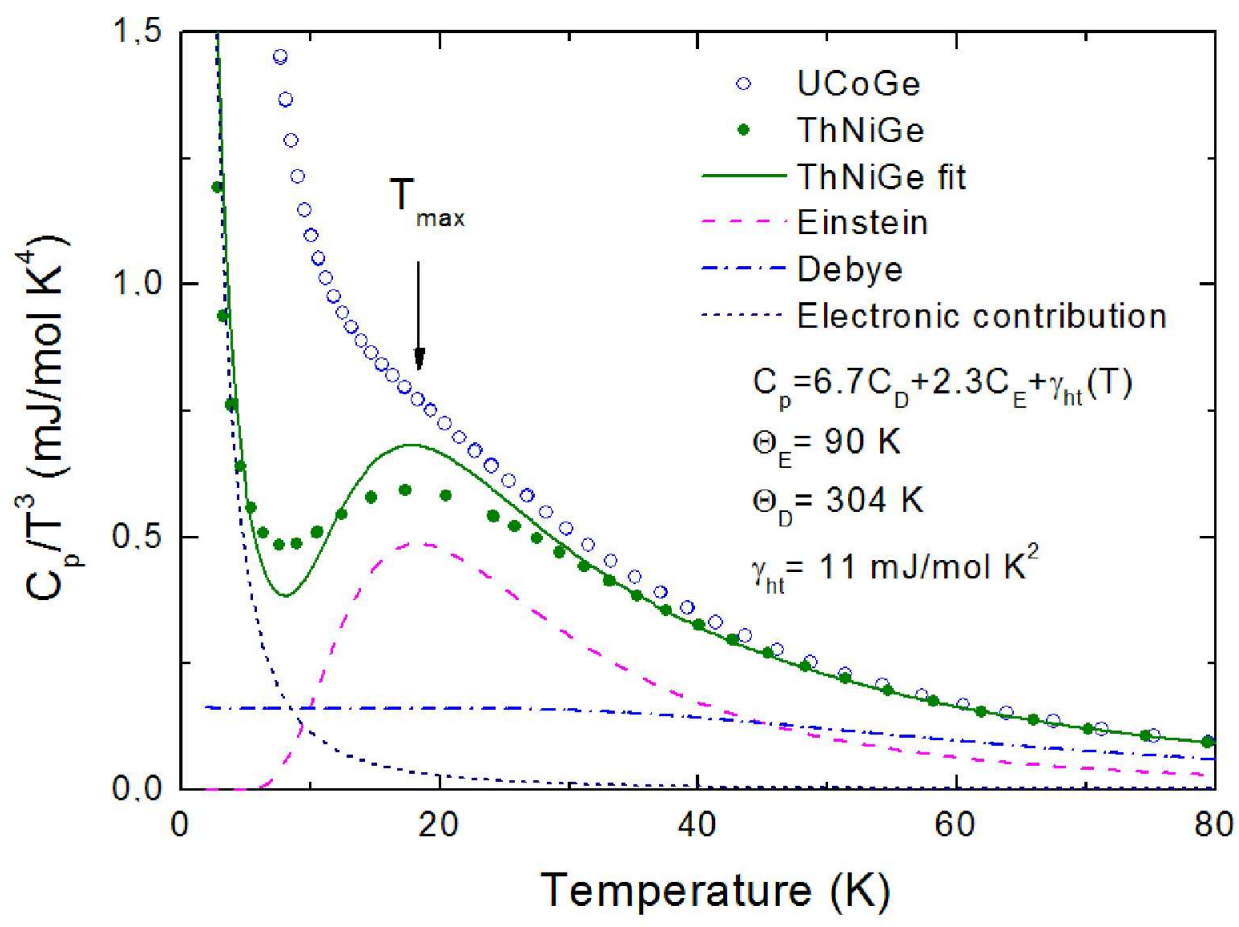

$344 \times 259 \mathrm{~mm}(600 \times 600$ DPI $)$ 